\title{
Characteristics of Incompressible Free Shear Flows and Implications for Turbulence Modeling *
}

\author{
Bernhard Eisfeld ${ }^{\dagger}$ \\ German Aerospace Center (DLR), Lilienthalplatz 7, D-38108 Braunschweig, Germany
}

It is shown that in self-preserving incompressible free shear flows governed by the boundarylayer equations a region must exist in which the Reynolds stress anisotropies are constant. The theoretical result is confirmed by an analysis of well-established experimental data for the plane jet, the axisymmetric jet and the plane mixing layer. The values of the corresponding Reynolds-stress anisotropies are determined, revealing differences between the corresponding eigensystems of these flows. While the shape of the corresponding tensor surfaces is similar, the corresponding principal axes have varying inclinations with respect to the flow-aligned coordinate system. Hence, the shear-stress anisotropy differs even in case of almost identical eigenvalues or invariants of the anisotropy tensor. Numerical predictions can be improved by a calibration of the pressure-strain correlation of a Reynolds-stress model using the observed anisotropies in the constant layer. For an industrial application, this will require an automatic adjustment of model coefficients according to the local flow type, although a self-adaptive model, that can switch automatically between the respective sets of tailored coefficients for each local flow type, is still being sought.

\section{Nomenclature}

$b_{i j} \quad$ Component of the Reynolds-stress anisotropy tensor

$\Delta b_{i j} \quad$ Uncertainty in component of the Reynolds-stress anisotropy tensor

$C_{i} \quad$ Coefficients of pressure-strain correlation

$C_{i}^{*} \quad$ Coefficients of pressure-strain correlation

$F_{i j} \quad$ Component of non-dimensional pressure-strain correlation

$f(\eta) \quad$ Profile function of mean velocity

$G_{x y}(\eta) \quad$ Scaled profile function of specific Reynolds-shear stress

$g_{i j}(\eta) \quad$ Profile function of specific Reynolds stress

$h \quad$ Height of orifice, $m$

$h(\eta) \quad$ Profile function of terms in Reynolds-stress equation

\footnotetext{
*Presented as Paper 2019-2962 at the AIAA Aviation Forum, Dallas, TX, 17-21 June 2019

${ }^{\dagger}$ Research Scientist, Dept. $\mathrm{C}^{2} \mathrm{~A}^{2} \mathrm{~S}^{2} \mathrm{E}$, Institute of Aerodynamics and Flow Technology.
} 


\begin{tabular}{|c|c|}
\hline$I_{b}$ & First invariant of Reynolds-stress anisotropy tensor \\
\hline$I I_{b}$ & Second invariant of Reynolds-stress anisotropy tensor \\
\hline$I I I_{b}$ & Third invariant of Reynolds-stress anisotropy tensor \\
\hline$k$ & Specific kinetic turbulence energy, $\mathrm{m}^{2} / \mathrm{s}^{2}$ \\
\hline$\ell(x)$ & Characteristic length scale, $\mathrm{m}$ \\
\hline$P^{(k)}$ & Production of $k, \mathrm{~m}^{2} / \mathrm{s}^{3}$ \\
\hline$P_{i j}$ & Component of Reynolds stress production tensor, $\mathrm{m}^{2} / \mathrm{s}^{3}$ \\
\hline$P_{s}$ & Scaling function of Reynolds stress production tensor, $\mathrm{m}^{2} / \mathrm{s}^{3}$ \\
\hline$R_{i j}$ & Component of specific Reynolds stress tensor, $\mathrm{m}^{2} / \mathrm{s}^{2}$ \\
\hline$R_{s}$ & Scaling function of specific Reynolds stresses, $\mathrm{m}^{2} / \mathrm{s}^{2}$ \\
\hline$r$ & Velocity ratio \\
\hline$r$ & Radial coordinate, $\mathrm{m}$ \\
\hline$r_{1 / 2}$ & Half-width of axisymmetric jet, $m$ \\
\hline$\widehat{S}$ & Spreading rate of jets \\
\hline$\widehat{S}_{\omega}$ & Spreading rate of mixing layer \\
\hline$S_{i j}$ & Component of strain rate tensor, $1 / \mathrm{s}$ \\
\hline$U$ & Streamwise velocity component, $\mathrm{m} / \mathrm{s}$ \\
\hline$U_{i}$ & Component of velocity vector, $\mathrm{m} / \mathrm{s}$ \\
\hline$U_{m}$ & Mean value of bounding mixing-layer velocities, m/s \\
\hline$U_{\max }$ & Maximum velocity in mixing layer, $\mathrm{m} / \mathrm{s}$ \\
\hline$U_{\min }$ & Minimum velocity in mixing layer, $\mathrm{m} / \mathrm{s}$ \\
\hline$U_{s}$ & Scaling function of mean velocity, $\mathrm{m} / \mathrm{s}$ \\
\hline$U_{z, 0}$ & Centerline velocity of axisymmetric jet, $\mathrm{m} / \mathrm{s}$ \\
\hline$U_{0}$ & Centerline velocity of plane jet, $\mathrm{m} / \mathrm{s}$ \\
\hline$\Delta U_{\max }$ & Local maximum velocity difference, $\mathrm{m} / \mathrm{s}$ \\
\hline$u_{i}^{\prime}$ & Component of fluctuating velocity, $\mathrm{m} / \mathrm{s}$ \\
\hline$W_{i j}$ & Component of rotation tensor, $1 / \mathrm{s}$ \\
\hline$x$ & Streamwise Cartesian coordinate, $\mathrm{m}$ \\
\hline$x_{i}$ & Cartesian coordinate, $\mathrm{m}$ \\
\hline$x_{i}^{(k)}$ & Coordinate of $k$ th eigenvector \\
\hline$y$ & Normal Cartesian coordinate, $\mathrm{m}$ \\
\hline$y_{1 / 2}$ & Half-width of plane jet, $\mathrm{m}$ \\
\hline
\end{tabular}


Axial coordinate, streamwise coordinate of axisymmetric jet, $\mathrm{m}$

\begin{tabular}{ll} 
Symbols & \\
$\beta_{i j}$ & Coefficient of corresponding Reynolds-stress component \\
$\delta_{i j}$ & Kronecker symbol \\
$\delta_{\omega}$ & Vorticity thickness, $\mathrm{m}$ \\
$\epsilon$ & Isotropic dissipation rate, $\mathrm{m}^{2} / \mathrm{s}^{3}$ \\
$\epsilon_{i j}$ & Cartesian component of dissipation rate tensor, $\mathrm{m}^{2} / \mathrm{s}^{3}$ \\
$\zeta$ & Scaled nondimensional normal coordinate \\
$\eta$ & Nondimensional normal coordinate \\
$\eta_{1 / 2}$ & Nondimensional jet half-width \\
$\lambda^{(A)}$ & Eigenvalue of tensor $A$ \\
$v$ & Kinematic viscosity, $\mathrm{m}^{2} / \mathrm{s}$ \\
$v_{t}$ & Turbulent viscosity, $\mathrm{m}^{2} / \mathrm{s}$ \\
$\Pi_{i j}$ & Component of pressure-strain correlation, $\mathrm{m}^{2} / \mathrm{s}^{3}$ \\
$\sigma(r)$ & Spreading parameter of mixing layer \\
$\sigma_{0}$ & Spreading parameter of half jet \\
$\sigma_{i j}$ & Circumferential coordinate \\
\hline &
\end{tabular}

Subscripts

av Average

exp Experimental value

$\max \quad$ Maximum

min Minimum

\section{Introduction}

Methods based on the Reynolds-averaged Navier-Stokes equations (RANS) are the backbone of numerical flow simulations in the aeronautical industry. While considered reliable for attached boundary layer flow, the accuracy of predictions is observed to degrade not only in case of separated flows [1], but even for simple free shear flows. An example is the so-called round-jet/plane-jet anomaly [2], where RANS-models often predict the spreading rate of a 
round jet to be greater than that of a plane jet, whereas experimental data show the opposite trend.

One reason for such failure might be found in the presence of large-scale coherent structures that have been observed by Brown and Roshko [3, 4], in addition to the small-scale turbulence in free shear flows. Since these coherent structures cannot be represented by an approach based on averaging, RANS-methods may not be suited for predicting free shear flows. In this view, any agreement of predictions with experimental data for free shear flows would be either fortuitous or due to non-physical fixes.

This position is contrasted by the results of a theoretical analysis by Görtler [5] who provides analytical solutions of the averaged boundary-layer equations for different incompressible free shear flows. Following a suggestion by Prandtl [6], these solutions are based on the assumption of a constant turbulent viscosity for the Reynolds-shear stress in each cross section. As shown in Fig. 1 these solutions are in remarkable agreement with classical experimental data for an incompressible plane jet, for an incompressible axisymmetric jet and for an incompressible plane mixing layer.

This agreement implies that the RANS-approach generally allows for accurately predicting at least incompressible free shear flows and that observed deviations must therefore be due to deficiencies of the particular models. In order to understand the reasons for such model deficiencies the common characteristics as well as the differences between incompressible plane and axisymmetric jets and plane mixing layers are subsequently investigated. A major focus is on the anisotropy of the Reynolds stresses, which appears to be constant in part of the respective shear layer. The values determined vary between the different flows, giving rise to different eigensystems. Even for almost identical eigenvalues (or invariants, respectively), the orientation of the principal axes of the anisotropy tensor and, thus, the Reynolds-stress tensor may differ, causing different levels of Reynolds-shear stress in a flow-aligned coordinate system.

The relevance of the constant anisotropy layer for turbulence modeling is demonstrated by recalibrating a Reynoldsstress model (RSM) according to the experimental anisotropies found in a plane mixing layer. With this modification, the agreement of predicted and experimental Reynolds stresses is clearly improved for this flow.

\section{Self-Similarity of Incompressible Jets and Mixing Layers}

Incompressible turbulent jets and mixing layers have long been studied, experimentally as well as theoretically. Early investigations, e. g. by Förthmann [7] for the plane jet, by Corrsin [8] for the axisymmetric jet and by Liepmann and Laufer [9] for the plane mixing layer, revealed a state of self-preservation, i. e. experimental results for the mean velocity $U$ collapse on a single profile, when scaled by the local maximum velocity difference $\Delta U_{\max }$ across the shear layer and a characteristic length scale $\ell$. For jets, a suitable length scale is the half width, $y_{1 / 2}$ for planar flow or $r_{1 / 2}$ for axisymmetric flow, i.e. the distance from the centerline, where half of the maximum velocity difference is reached. 
For plane mixing layers, the vorticity thickness

$$
\delta_{\omega}=\frac{\Delta U_{\max }}{\left.\frac{d U}{d y}\right|_{\max }}
$$

is an appropriate choice.

Similarly, the profiles of the specific Reynolds stresses $R_{i j}=\overline{u_{i}^{\prime} u_{j}^{\prime}}$, where $u_{i}^{\prime}$ denotes the components of the fluctuating velocity, are observed to collapse, when scaled by the square of the local maximum velocity difference $\left(\Delta U_{\max }\right)^{2}$.

Subsequent experimental investigations employed refined measurement techniques and confirmed the self-preservation of the turbulence. Examples are the experimental work of Bradbury [10], Heskestad [11] and Gutmark and Wygnanski [12] for the plane jet, of Antonia and Bilger [13], Wygnanski and Fiedler [14] and Hussein et al. [15] for the axisymmetric jet and of Wygnanski and Fiedler [16], Tavoularis and Corrsin [17], Delville et al. [18], Bell and Mehta [19] and Mehta [20] for the plane mixing layer. Many of these experiments have also been considered as reference cases for the validation of Large-Eddy Simulations [21], where the experimental data are publicly available[22].

After the major characteristics of the self-preserving region had been established, research concentrated on different topics. As documented by Ball et al. [23] in their review, for the axisymmetric turbulent jet there has been an increased interest in the intermediate region where the flow transitions to its self-preserving state. In particular, the influence of the inflow conditions on the mixing transition has been studied intensively [24-29]. Another focus has been the determination of coherent structures and vortices near the orifice [30], in the transition region [31-34] and in the self-preserving region [35, 36]. Self-preservation has been demonstrated also for two-point correlations [37] and structure functions [38] in incompressible turbulent axisymmetric jets.

Except Ferdman et al. [24], none of these more recent investigations on the incompressible axisymmetric turbulent jet provides complete profile data of all Reynolds stresses at distances greater than 70 orifice diameters downstream of the inlet, which is required for establishing self-preservation [23, 31]. In the cross-plots of experimental profile data by Ball et al. [23] the experiment by Hussein et al. [15] appears to be the most recent one providing such a complete data set at large enough distance from the orifice. Also, Refs. [28, 29, 31, 33, 37, 38], refer to the experiment of Hussein et al. [15] for comparison with their own data. For this reason, the data of Hussein et al. [15] are used in this paper for reference for the incompressible axisymmetric jet.

The incompressible plane turbulent jet has gained less attention in research than its axisymmetric counterpart [39, 40]. Similar to the axisymmetric jet, recent investigations have focused on the influence of the initial flow conditions at the orifice, e.g. Refs [41, 42], and on coherent structures, e.g. Refs. [43, 44]. This might be why Matsubara et al. [44] only recently denote the experimental data by Gutmark and Wygnanski [12] as a standard reference. The experiments by Gordeyev and Thomas [43] and Cafiero and Vassilicos [45] suggested alternatively by 
Matsubara et al. [44], however, do not provide profiles of the spanwise Reynolds-normal stress and do not reach as far downstream as the experiment by Gutmark and Wygnanski [12]. For this reason, the latter data are used in this paper for reference for the incompressible plane jet.

Experimental studies of the incompressible plane mixing layer show a variety of research interests. Naka et al. [46] measured pressure and velocity simultaneously, but restricted themselves to the near-field, upstream of self-preservation. A number of groups employed Particle Image Velocimetry (PIV), where Li et al [47] measured simultaneously with PIV and hot wire anemometry (HWA) for determining the optimum mode of operation. Guo et al. [48] used PIV for investigating the influence of the velocity ratio on the near field in the developing mixing layer. The group of Buxton and co-workers [49-51] studied the convection speed of fluctuations of different scales and their interaction in the far-field of an incompressible mixing layer by means of PIV. However, none of these investigations reports complete profile data of all Reynolds stresses in the self-preserving region of the mixing layer.

According to Loucks and Wallace [52] there are many experiments on the incompressible plane mixing layer, providing two velocity components and the corresponding Reynolds stresses. In contrast Loucks and Wallace [52] as well as Li et al. [53] also provide data for the spanwise velocity component and Reynolds-normal stress, however, in only very few points across the mixing layer. Moreover, they report excellent agreement with the data by Bell and Mehta [19] confirming the relevance of the test cases in the AGARD database for LES validation [21, 22].

The most important mixing layer test case of this collection is probably the experiment by Delville et al. [18] that is also included in the ERCOFTAC data base [54] as test case C.34 and on the NASA Turbulence Modeling Resource website [55] as test case 2DML. There have been follow-on experiments by the same group [56, 57] that, however, did not find their way into these databases. Indeed, the experiment in Ref. [56] has a rather coarse resolution across the mixing layer, and the comparison of maximum Reynolds-stress ratios in Ref. [57] shows significantly lower streamwise fluctuations than reported by others. For this reason, the experimental data in the AGARD database [21, 22], particularly the data by Delville et al. [18] are used in this paper for reference.

Generally, it has been found that, for observing a state of self-preservation, a sufficiently high local Reynolds number is required. In particular, self-preservation of the Reynolds stresses requires higher Reynolds numbers than self-preservation of the mean velocity. According to Dimotakis [58], a local Reynolds number of $R e=1 \cdot 10^{4}-$ $2 \cdot 10^{4}$ is required for obtaining a fully established self-preserving turbulent flow. Despite the considerable progress in computational methods and available power, this condition still poses a significant problem for scale-resolving simulations particularly of spatially developing flows, e.g. Refs. [59, 60]. Note that with incompressible plane jets and mixing layers the local Reynolds number increases with the downstream distance from their respective origin.

The experimental observations imply that the mean velocity and the specific Reynolds stresses can be described in 
non-dimensional form by

$$
\begin{aligned}
\frac{U}{\Delta U_{\max }(x)} & =f(\eta), \\
\frac{R_{i j}}{\left[\Delta U_{\max }(x)\right]^{2}} & =g_{i j}(\eta),
\end{aligned}
$$

in which $x$ is the streamwise coordinate,

$$
\eta=\frac{y}{\ell(x)}
$$

is the non-dimensional normal coordinate, and $f(\eta)$ and $g_{i j}(\eta)$ are non-dimensional profile functions.

As mentioned in the Introduction, Prandtl [6] suggested assuming a constant turbulent viscosity across the respective shear layer for describing the Reynolds-shear stress, being aware that this is incorrect towards the layer's edge. Based on this assumption, Görtler [5] elaborated self-similar solutions of the incompressible boundary-layer equations for the mean velocity profile of the plane jet and the plane mixing layer. The self-similar solution for the incompressible axisymmetric turbulent jet can then be derived, following the procedure of Schlichting [61] for the laminar case. These self-similar solutions follow the experimentally deduced form of the non-dimensional velocity profile, Eq. (2), from which the profile of the non-dimensional specific Reynolds shear stress follows in agreement with Eq. (3), and, as shown in Fig. 1] appear to be in good agreement with experimental data in the central part of the respective flow.

Note that Görtler [5] provides the mixing-layer solution for an arbitrary velocity ratio of the two streams, $r=$ $U_{\min } / U_{\max }$, only numerically in terms of a series expansion. Nevertheless, an analytical solution can be obtained for the limit of vanishing velocity difference, i. e. $r \rightarrow 1$, which is also denoted as the (incompressible) temporal mixing layer [62].This analytical solution is still a good approximation of the incompressible plane mixing layer flow at $r<1$, as is demonstrated by the comparison in Fig. 1. However, due to its more approximate character with decreasing velocity ratio $r$, the deviation from the experimental data is expected to be more rapid towards the edges of the mixing layer compared to the jet flows. This seems to be confirmed particularly by the Reynolds-shear stress profile.

The theoretical solutions according to Görtler [5] are summarized in the Appendix.

\section{Reynolds-Stress Anisotropy}

Within the RANS-concept, turbulence is described by the specific Reynolds stresses $R_{i j}=\overline{u_{i}^{\prime} u_{j}^{\prime}}$, correlating fluctuations of the velocity components $u_{i}^{\prime}$. Their non-dimensional representation are the corresponding anisotropies

$$
b_{i j}=\frac{R_{i j}}{2 k}-\frac{1}{3} \delta_{i j}
$$

in which $k=R_{i i} / 2$ denotes the specific kinetic turbulence energy and $\delta_{i j}$ represents the Kronecker symbol. 
The anisotropy tensor is symmetric, $b_{i j}=b_{j i}$, and traceless, $b_{i i}=0$. Its characteristic equation reads

$$
-\left(\lambda^{(b)}\right)^{3}+I_{b}\left(\lambda^{(b)}\right)^{2}-I I_{b} \lambda^{(b)}+I I I_{b}=0
$$

in which $\lambda^{(b)}$ refers to the eigenvalues of the anisotropy tensor and

$$
\begin{aligned}
I_{b} & =b_{i i}=0, \\
I I_{b} & =-\frac{1}{2} b_{i j} b_{i j}, \\
I I I_{b} & =\frac{1}{3} b_{i j} b_{j k} b_{k i}
\end{aligned}
$$

are the invariants, where the second and third invariant are often used for characterising the turbulence state [63]. Note that the invariants of the anisotropy tensor can be expressed in terms of its eigenvalues according to

$$
\begin{aligned}
I_{b} & =\lambda_{1}^{(b)}+\lambda_{2}^{(b)}+\lambda_{3}^{(b)}=0, \\
I I_{b} & =\lambda_{1}^{(b)} \lambda_{2}^{(b)}+\lambda_{1}^{(b)} \lambda_{3}^{(b)}+\lambda_{2}^{(b)} \lambda_{3}^{(b)}, \\
I I I_{b} & =\lambda_{1}^{(b)} \lambda_{2}^{(b)} \lambda_{3}^{(b)} .
\end{aligned}
$$

Hence, invariants and eigenvalues can alternatively be used for characterising the properties of the Reynolds-stress anisotropy tensor. Subsequently, these properties are investigated in incompressible jets and mixing layers.

\section{III.A Theoretical Considerations}

Jets and mixing layers are characterised by a predominant mean-flow direction and a predominant velocity gradient normal to this direction that is limited to a thin region around the center of the flow. For this reason, the boundary layer assumptions, originally derived for wall-bounded flows at high local Reynolds number, apply to these flows [62, 64], which has already been implicitly assumed by Görtler [5]. For simplicity, a flow-aligned Cartesian coordinate system is used in the following with $x$ in the direction of the predominant mean velocity $U, y$ in the normal direction along the predominant mean-velocity gradient $\partial U / \partial y$ and $z$ in the spanwise direction. The results can be directly transferred to axisymmetric flows, using cylindrical coordinates with $z$ in the direction of the predominant velocity $U_{z}, r$ in the radial direction of the predominant velocity gradient $\partial U_{z} / \partial r$ and $\phi$ in the circumferential direction. According to the incompressibility assumption, the density and the viscosity of the fluid are taken as constant.

Following the arguments of Rotta [65] and Hinze [64], at very high local Reynolds number there should exist a region, where production $P_{i j}$, dissipation $\epsilon_{i j}$ and the pressure-strain correlation $\Pi_{i j}$ of the Reynolds-stress transport 
equation are in equilibrium according to

$$
P_{i j}-\epsilon_{i j}+\Pi_{i j}=0
$$

This equation has been used e.g., by Launder, Reece and Rodi [66] (LRR) for calibrating the pressure-strain correlation of their Reynolds-stress model.

In incompressible flow the pressure-strain correlation is traceless [67] so that, from Eq. [13], follows the equilibrium condition for the specific kinetic turbulence energy,

$$
P^{(k)}=\epsilon,
$$

in which $P^{(k)}=P_{k k} / 2$ denotes the production of specific kinetic turbulence energy and $\epsilon=\epsilon_{k k} / 2$ the isotropic dissipation rate. Equation (14) is fundamental for the calibration of turbulence models to the log-law in incompressible boundary layers [2], which is subject to the same assumptions. Experimental data on the balance of the specific kinetic turbulence energy confirm the existence of small regions compared to the shear-layer width, where $P^{(k)} \approx \epsilon$ e. g., in the incompressible plane jet [10-12], in the incompressible axisymmetric jet [15, 16] and in the incompressible plane mixing layer [14].

According to the boundary-layer assumptions, the only non-zero normal-stress production term is in the streamwise direction so that

$$
\begin{aligned}
& P_{x x}=-2 R_{x y} \frac{\partial U}{\partial y}, \\
& P_{y y}=0 \\
& P_{z z}=0
\end{aligned}
$$

and hence

$$
P^{(k)}=\frac{1}{2}\left(P_{x x}+P_{y y}+P_{z z}\right)=-R_{x y} \frac{\partial U}{\partial y} .
$$

Note that, on the jet-centerline, the symmetry conditions require $\left.P^{(k)}\right|_{c l}=0$, whereas the dissipation rate does not vanish there, $\left.\epsilon\right|_{c l} \neq 0$. For this reason, the equilibrium condition (14) cannot be supposed to hold on the centerline of symmetric jets. As will be shown below, this is indeed confirmed by the experimental data for the incompressible plane jet [10-12] and the incompressible axisymmetric jet [15, 16].

At sufficiently high Reynolds number and far enough away from walls or singularities in the flow field, the turbulence becomes locally isotropic [62, 65], being associated with an isotropic dissipation tensor [62, 65], i. e.

$$
\epsilon_{i j}=\frac{2}{3} \epsilon \delta_{i j}
$$


Corrsin [68] has derived the condition for local isotropy as

$$
\sqrt{\frac{v}{\epsilon}} \frac{\partial U}{\partial y} \ll 1
$$

which has been experimentally confirmed by Saddoughi and Veeravalli [69]. Introducing the condition of turbulent equilibrium (14), this corresponds to

$$
v \frac{\partial U}{\partial y} \ll-R_{x y}
$$

indicating that local isotropy and hence isotropy of the dissipation tensor requires the viscous shear stress to be negligible compared to the Reynolds-shear stress. Indeed, this is part of the assumptions made by Görtler [5] in his derivation of self-similar solutions.

Now assume that the mean velocity $U$ and the specific Reynolds-shear stress $R_{x y}$ are self-similar so that, according to George [70], they can be written as

$$
\begin{aligned}
U & =U_{s}(x) f(\eta), \\
R_{x y} & =R_{s}(x) g_{x y}(\eta),
\end{aligned}
$$

where $U_{S}(x)$ and $R_{S}(x)$ are scaling functions of the mean velocity and the specific Reynolds-shear stress, respectively, and $f(\eta)$ and $g_{x y}(\eta)$ are the corresponding non-dimensional profile functions, depending on the non-dimensional normal coordinate $\eta$ defined in Eq. (4). Note that, according to Eq. (2) and Eq. (3), it is typically assumed that $U_{S}(x)=\Delta U_{\max }(x)$ and $R_{S}(x)=U_{S}^{2}(x)$. Nevertheless, the following analysis is independent of any assumption on the scaling functions and their definition.

If the mean velocity $U$ and the specific Reynolds-shear stress $R_{x y}$ are self-similar according to Eqs. (22) and (23), then the $k$-production term, Eq. (18), and hence the isotropic dissipation rate also become self-similar according to

$$
P^{(k)}=P_{S}(x) h(\eta)=\epsilon
$$

with

$$
\begin{aligned}
P_{S}(x) & =-\frac{U_{S}(x) R_{S}(x)}{\ell(x)} \\
h(\eta) & =g_{x y}(\eta) \frac{d f}{d \eta}
\end{aligned}
$$


Since the dissipation is supposed to be isotropic,

$$
\epsilon_{x x}=\epsilon_{y y}=\epsilon_{z z}=\frac{2}{3} P_{s}(x) h(\eta)
$$

and the normal-stress components of the pressure-strain correlation are obtained from the equilibrium condition (13) as

$$
\begin{aligned}
\Pi_{x x} & =-\frac{4}{3} P_{s}(x) h(\eta), \\
\Pi_{y y} & =\frac{2}{3} P_{s}(x) h(\eta), \\
\Pi_{z z} & =\frac{2}{3} P_{s}(x) h(\eta) .
\end{aligned}
$$

Obviously, the conditions of self-similarity and turbulent equilibrium require the terms of production, dissipation and the pressure-strain correlation all to follow an identical profile function $h(\eta)$.

Following the arguments of Durbin and Petterson Reif [71], the pressure-strain correlation must have the general functional form

$$
\Pi_{i j}=\epsilon F_{i j}\left[b_{i j}, \frac{k}{\epsilon} \frac{\partial U_{i}}{\partial x_{j}}\right]
$$

in which $F_{i j}$ is non-dimensional. Clearly, if $\Pi_{i j}$ and $\epsilon$ follow the same profile function $h(\eta)$, the tensorial function $F_{i j}$ must be constant, implying that its arguments are constant. From the first argument it follows

$$
b_{i j}=\text { const }
$$

In the second argument the components of the velocity gradient tensor can be replaced by their dominant component, $\frac{\partial U}{\partial y}$, according to the boundary-layer assumptions. Therefore, the second argument becomes

$$
\frac{k}{\epsilon} \frac{\partial U_{i}}{\partial x_{j}} \approx \frac{k}{\epsilon} \frac{\partial U}{\partial y}=\frac{R_{x y} \frac{\partial U}{\partial y}}{\epsilon} \frac{k}{R_{x y}}=-\frac{P^{(k)}}{\epsilon} \frac{k}{R_{x y}}=-\frac{1}{2 b_{x y}}=\text { const. }
$$

which is obviously compatible with the condition in Eq. (32).

Thus one can conclude that any self-preserving flow that is governed by the incompressible boundary-layer equations will exhibit a layer that is in turbulent equilibrium, Eq. [13, and in which the Reynolds-stress anisotropies $b_{i j}$ all are constant. These assumptions hold for the plane and axisymmetric jet as well as for plane mixing layers. Note that, due to the symmetry condition, the region of constant Reynolds-stress anisotropies is not supposed to be found on the centerline of the jets.

The above result is related to the findings of Abid and Speziale [72], who, along a similar line of arguments, show 
constant Reynolds-stress anisotropy in the log-layer of incompressible turbulent channel flow and in homogeneous shear flow. It also complies with the Bradshaw hypothesis [73] assuming $\left|b_{x y}\right| \approx 0.150=$ const. in boundary layers. Furthermore, Dairay et al. [74] required the assumption of constant Reynolds-stress anisotropies, in order to derive their non-equilibrium dissipation scaling in incompressible self-similar axisymmetric wakes. There is also a relationship to the balance between anisotropy production and the rapid redistribution term that, according to Rapid Distortion Theory, holds in the limit of time-independent anisotropy [75].

\section{III.B Experimental Confirmation}

Experimental confirmation of the constant anisotropy hypothesis is hampered by the fact that the velocity fluctuations in three orthogonal directions are required at the same position in space, in order to provide the specific kinetic turbulence energy needed for non-dimensionalisation. Many of the well-established experiments have been carried out employing hot-wire anemometry with single or cross-wire probes, necessitating repeated traverses across the shear layer with the probe rotated to obtain all three velocity components. Unfortunately, the probe positions have not always been identical during the repetition, introducing an additional uncertainty.

In order to reduce this uncertainty and to use data obtained at different positions along the traverse, the respective theoretical descriptions based on the assumption of constant turbulent viscosity are employed. This procedure is justified by the agreement of the theoretical mean velocity profiles with experimental data in the central part of the different incompressible shear layers, shown in the left column of Fig. 1 .

The specific Reynolds shear stress must then follow an associated profile function

$$
g_{x y}(\eta)=\beta_{x y} G_{x y}(\eta)
$$

that can be directly derived from the respective velocity profile $f(\eta)$. The values of the coefficient $\beta_{x y}$ are constant and have been determined by the author such that best agreement with the data is obtained in the central part of the respective flow, cf. Ref. [76]. The corresponding Reynolds-shear stress profiles are shown in the right column of Fig.

1 Clearly, there is some scatter in the experimental data, nevertheless the agreement with the theoretical curves seems to confirm the theoretical descriptions in the central part of the respective flows.

Constant Reynolds-stress anisotropy requires all non-dimensional specific Reynolds-normal stresses to follow the same profile function, differing only by some constant scaling factor. In particular, all Reynolds stresses can be expressed in terms of the profile function for the non-dimensional specific Reynolds-shear stress, $G_{x y}$, according to

$$
\frac{R_{i j}(x, \eta)}{R_{s}(x)}=\beta_{i j} G_{x y}(\eta)
$$


in which $\beta_{i j}$ represents the scaling factor for the individual component. Given the theoretical profile function for the specific Reynolds-shear stress, $G_{x y}(\eta)$, the respective coefficients are obtained from experimental data by

$$
\beta_{i j}=\frac{\left[R_{i j}(x, \eta) / R_{s}(x)\right]_{e x p}}{G_{x y}(\eta)}
$$

in which $\left[R_{i j}(x, \eta) / R_{S}(x)\right]_{\text {exp }}$ refers to any measured non-dimensional specific Reynolds-stress component. Typically, experimental data are scaled by the square of the maximum velocity difference in a cross-section, $R_{S}(x)=\left[\Delta U_{\max }(x)\right]^{2}$.

Thus, a region of constant Reynolds-stress anisotropies would be indicated by a region of constant coefficients $\beta_{i j}$. Note that, in case of the axisymmetric jet, $G_{x y}(\eta)$ is replaced by $G_{r z}(\eta)$.

Figures 2, 3and 4 show the profiles of the coefficients $\beta_{i j}$ obtained from an analysis of the experiments of Gutmark and Wygnanski [12] for the incompressible plane jet, of Hussein et al. [15] for the incompressible axisymmetric jet and of Delville et al. [18] for an incompressible plane mixing layer at velocity ratio $r=0.54$. Indeed, all cases show a region where the coefficients $\beta_{i j}$ are approximately constant. Nevertheless its extent and pronounciation depends on the respective flow and the Reynolds-stress component and might be influenced by the experimental set-up (Fig. 3) or measurement position, i. e. local Reynolds number (Fig. 44). In agreement with the theoretical consideration that Eq. (14) does not hold on the centerline of symmetric jets, in the jet flows the indicated region occurs at some distance from the centerline and extends approximately from the region around the theoretical position of the maximum specific Reynolds-shear stress to the half-width or slightly beyond. In the plane mixing layer it is located around the dividing streamline, covering at least $20-40 \%$ of the vorticity thickness $\delta_{\omega}$ in both directions.

Thus, the experimental data generally confirm the theoretically deduced layer of constant Reynolds-stress anisotropies in incompressible turbulent jets and the incompressible turbulent plane mixing layer. Note that recent experiments by Kim et al. [77] confirm the existence of a constant-anisotropy layer even in compressible mixing layers.

\section{Turbulence Structure}

\section{IV.A Reynolds-Stress Anisotropy}

As shown by Pope [62], only the anisotropic part of the specific Reynolds-stress components is effective in transporting momentum. Hence the accuracy of turbulence models depends on their ability to predict the Reynoldsstress anisotropies $b_{i j}$ correctly, in particular that of the Reynolds-shear stress, $b_{x y}$ or $b_{r z}$. For this reason, the Reynolds-stress anisotropies of the investigated flows are deduced from the results for the coefficients $\beta_{i j}$ in the constant region.

According to Eq. (35), in this region the specific Reynolds stresses all follow the same profile function $G_{x y}(\eta)$, differing only in the value of the respective coefficient $\beta_{i j}$. Hence, the definition of the Reynolds-stress anisotropies, 
Eq. (5), yields

$$
b_{i j}=\frac{\beta_{i j}}{\beta_{k k}}-\frac{1}{3} \delta_{i j}
$$

allowing to compute the Reynolds-stress anisotropies directly from the values of the coefficients $\beta_{i j}$ in the constant region obtained from the experimental data.

In order to reduce the uncertainty in the data, the coefficients $\beta_{i j}$ are averaged over the respective constant region. Since Gutmark and Wygnanski [12] and Delville et al. [18] provide their data at different downstream positions, the averaging has been carried out for each data set individually. In contrast, Hussein et al. [15] provide data only at one position, for which they nevertheless checked that self-preservation has been achieved.

Figure 5 shows the streamwise development of the averaged coefficients $\left(\beta_{i j}\right)_{a v}$ for the plane-jet data of Gutmark and Wygnanski [12] and for the plane mixing-layer data of Delville et al. [18], in which also data from further upstream positions have been included. As one can see, the plane-jet results seem to be fairly independent of the downstream position, confirming that self-preservation has been achieved, whereas the mixing-layer results still exhibit some variation. In contrast, the standard deviation of the plane-jet data given in Tab. 1 is generally larger than that of the plane mixing-layer data.

The largest relative uncertainties are observed for the Reynolds-normal stress in the direction of the predominant mean-velocity gradient, where for the plane-jet data at the most downstream position the standard deviation $\sigma_{y y}$ is approximately $7 \%$ of the mean value $\left(\beta_{y y}\right)_{a v}$. For all other components the standard deviation is of the order of $2 \%$ to $3.5 \%$ of the corresponding mean value. For the plane mixing-layer data it is of the order of $1 \%$ to $2 \%$ for all components.

Table 1 contains the averaged Reynolds-stress coefficients $\left(\beta_{i j}\right)_{a v}$ together with the associated standard deviations $\sigma_{i j}$ for the different flows. The values for the plane-jet experiment of Gutmark and Wygnanski [12] and the plane mixing-layer experiment of Delville et al. [18] refer to the respective most downstream position associated with the highest local Reynolds number. For the axisymmetric-jet experiment of Hussein et al. [15], the averages of the data obtained by a flying hot-wire and by Laser-Doppler anemometry are provided separately. Note the difference between the coefficients $\beta_{r r}$ and $\beta_{\phi \phi}$, indicating some deviation from the axisymmetry of the jet.

Table 2] contains the Reynolds-stress anisotropies $b_{i j}$ for the different flows inferred from the averaged coefficients $\left(\beta_{i j}\right)_{a v}$ in Table 1 according to Eq. (37). For comparison, the boundary-layer values according to the classical log-law assumption [2], $R_{x x}: R_{y y}: R_{z z}=4: 2: 3$, and the Bradshaw hypothesis [73] for the Reynolds-shear stress have been included.

For the axisymmetric jet, the values refer to the average results obtained from the flying hot-wire data and the Laser-Doppler anemometry data of Hussein et al. [15], where the values of $b_{r r}$ and $b_{\phi \phi}$ have been additionally averaged, in order to compensate for the observed deviation from axisymmetry. The $\Delta b_{i j}$ in Table 2 refer to an estimate of the 
Table 1 Averaged Reynolds-stress coefficients in the constant region.

\begin{tabular}{lcccccccc} 
Flow & $\left(\beta_{x x}\right)_{a v}$ & $\sigma_{x x}$ & $\left(\beta_{y y}\right)_{a v}$ & $\sigma_{y y}$ & $\left(\beta_{z z}\right)_{a v}$ & $\sigma_{z z}$ & $\left(\beta_{x y}\right)_{a v}$ & $\sigma_{x y}$ \\
\hline Plane jet [12] & 0.2519 & 0.00762 & 0.0832 & 0.00559 & 0.0941 & 0.00317 & 0.0628 & 0.00149 \\
Plane mixing layer [18] & 0.0274 & 0.00023 & 0.0168 & 0.00037 & 0.0229 & 0.00034 & 0.0116 & 0.00021 \\
& $\left(\beta_{z z}\right)_{a v}$ & $\sigma_{z z}$ & $\left(\beta_{r r}\right)_{a v}$ & $\sigma_{r r}$ & $\left(\beta_{\phi \phi}\right)_{a v}$ & $\sigma_{\phi \phi}$ & $\left(\beta_{r z}\right)_{a v}$ & $\sigma_{r z}$ \\
Axisymmetric jet [15], & 0.1394 & 0.01356 & 0.0750 & 0.00787 & 0.0778 & 0.00662 & 0.0387 & 0.00404 \\
$\begin{array}{l}\text { Flying Hot Wire } \\
\text { Axisymmetric jet [15] }\end{array}$ & 0.1409 & 0.01555 & 0.0765 & 0.00655 & 0.0843 & 0.00564 & 0.0393 & 0.00316 \\
Laser-Doppler & & & & & & & &
\end{tabular}

Table 2 Reynolds-stress anisotropies in the constant region.

\begin{tabular}{lcccccccc} 
Flow & $b_{x x}$ & $\Delta b_{x x}$ & $b_{y y}$ & $\Delta b_{y y}$ & $b_{z z}$ & $\Delta b_{z z}$ & $b_{x y}$ & $\Delta b_{x y}$ \\
\hline Boundary layer [2,73] & 0.111 & - & -0.111 & - & 0 & - & -0.150 & - \\
Plane jet [12] & 0.254 & 0.011 & -0.139 & 0.011 & -0.114 & 0.008 & 0.146 & 0.005 \\
Plane mixing layer [18] & 0.075 & 0.004 & -0.083 & 0.004 & 0.008 & 0.004 & 0.173 & 0.003 \\
Plane mixing layer [17, 19, 20] & 0.114 & 0.005 & -0.085 & 0.002 & -0.029 & 0.003 & 0.164 & 0.012 \\
& $b_{z z}$ & $\Delta b_{z z}$ & $b_{r r}$ & $\Delta b_{r r}$ & $b_{\phi \phi}$ & $\Delta b_{\phi \phi}$ & $b_{r z}$ & $\Delta b_{r z}$ \\
Axisymmetric jet [15] & 0.139 & 0.030 & -0.069 & 0.022 & -0.069 & 0.022 & 0.131 & 0.014
\end{tabular}

uncertainty associated with the averaging of the coefficients $\beta_{i j}$. They have been obtained from

$$
\Delta b_{i j}=\sqrt{\left(\left.\frac{\partial b_{i j}}{\partial \beta_{x x}}\right|_{a v} \sigma_{x x}\right)^{2}+\left(\left.\frac{\partial b_{i j}}{\partial \beta_{y y}}\right|_{a v} \sigma_{y y}\right)^{2}+\left(\left.\frac{\partial b_{i j}}{\partial \beta_{z z}}\right|_{a v} \sigma_{z z}\right)^{2}+\left(\left.\frac{\partial b_{i j}}{\partial \beta_{x y}}\right|_{a v} \sigma_{x y}\right)^{2}},
$$

where there is no summation on $i$ and $j$. The derivatives follow from Eq. [37), relating the Reynolds-stress anisotropies $b_{i j}$ to the coefficients $\beta_{i j}$. Note that Eq. (38) does not allow for a compensation of errors and thus yields a rather conservative estimate.

An additional cross-comparison of various incompressible mixing-layer data has revealed that the experimental results by Delville et al. [18] deviate from those obtained by Bell and Mehta [19], Mehta [20] and Tavoularis and Corrsin [17]. For this reason, an estimate of the anisotropies based on these data together with an estimate of the corresponding uncertainties has been added to Tab. 2. Details are given in Ref. [76].

Despite the estimated uncertainties in the data, the values in Tab. 2 indicate differences between the turbulence structures of the respective flows. 
Table 3 Characteristics of the anisotropy tensor in the constant region.

\begin{tabular}{lcccccc} 
Flow & \multicolumn{1}{c}{$I I_{b}$} & \multicolumn{1}{c}{$I I_{b}$} & $\lambda_{1}^{(b)}$ & \multicolumn{1}{c}{$\lambda_{2}^{(b)}$} & \multicolumn{1}{c}{$\lambda_{3}^{(b)}$} & \multicolumn{1}{c}{$\theta$} \\
\hline Boundary layer $[2,73]$ & -0.0348 & 0 & 0.187 & -0.187 & 0 & $-26.7^{o}$ \\
Plane jet [12] & -0.0698 & 0.00651 & 0.302 & -0.187 & -0.115 & $18.3^{o}$ \\
Axisymmetric jet [15] & -0.0317 & 0.00187 & 0.202 & -0.132 & -0.070 & $25.8^{o}$ \\
Plane mixing layer [18] & -0.0362 & -0.00029 & 0.186 & -0.194 & 0.008 & $32.8^{o}$ \\
Plane mixing layer [17, 19, 20] & -0.0374 & 0.00106 & 0.206 & -0.177 & -0.029 & $29.4^{o}$
\end{tabular}

\section{IV.B Invariants, Eigenvalues and Principal Axes}

For characterising the turbulence structure of the respective flows, the invariants of the anisotropy tensor have been computed according to Eqs. (8) and (9) together with the corresponding eigenvalues. The results are summarised in Tab. 3, where the third eigenvalue $\lambda_{3}^{(b)}$ follows from the condition of tracelessness. Note that the invariants and eigenvalues for the incompressible plane mixing layer, particularly when referring to the data by Delville et al. [18], are rather close to those for the classical boundary layer assumptions [2, 73], implying a similar turbulence structure in those flows.

This is confirmed by plotting the invariants of the respective flows into the invariant map shown in Fig. 6(left). Originally derived by Lumley [63], this graph represents the domain of physically possible turbulence states in terms of the invariants of the Reynolds-stress anisotropy tensor. As shown by the data in Tab. 3 the turbulence states of the incompressible boundary layer and the incompressible plane mixing layer, particularly when referring to the data of Delville et al. [18], are very close to one another, whereas the jet flows are somewhat different.

The eigenvalues, closely related to the invariants of the Reynolds-stress anisotropy tensor, allow for a geometrical interpretation of the associated Reynolds-stress tensor itself. Since the latter is positive semi-definite, it is associated with a tensor surface representing an ellipsoid. The lengths of its semi-axes are given by the square-root of the respective eigenvalues of the Reynolds-stress tensor that are obtained from the eigenvalues of the corresponding anisotropy tensor according to

$$
\lambda_{i}^{(R)}=2 k\left(\lambda_{i}^{(b)}+\frac{1}{3}\right)
$$

Since eigenvalues and invariants are interrelated, each location in the invariant map, Fig. 6, is thus associated with a particular aspect ratio, i. e. shape, of the corresponding ellipsoid.

However, in order to describe the anisotropy tensor completely, its principal axes are required to be defined by the respective eigenvectors $\vec{x}^{(k)}$. They follow from the anisotropy components in Tab. 2 and the corresponding eigenvalues in Tab. 3 for the constant region according to

$$
b_{i j} x_{j}^{(k)}=\lambda_{k}^{(b)} x_{i}^{(k)}, \quad k=1,2,3
$$


Since the flows under investigation are two-dimensional, it suffices to consider the eigensystem in the flow plane; the eigenvector $\vec{x}^{(3)}$ associated with the third eigenvalue $\lambda_{3}^{(b)}$ is oriented into the spanwise or circumferential direction, respectively.

Figure 6 (right) shows the principal axes of the anisotropy tensor and, hence, the Reynolds-stress tensor together with the associated ellipses for the different flows with respect to the flow-aligned coordinate system. The semi-axes of the ellipses have been scaled by the specific kinetic turbulence energy $k$, yielding

$$
\begin{aligned}
& x=\sqrt{\frac{\lambda_{1}^{(R)}}{k}} \cos \phi=\sqrt{2\left(\lambda_{1}^{(b)}+\frac{1}{3}\right)} \cos \phi, \\
& y=\sqrt{\frac{\lambda_{2}^{(R)}}{k}} \sin \phi,=\sqrt{2\left(\lambda_{2}^{(b)}+\frac{1}{3}\right)} \sin \phi, \quad 0 \leq \phi \leq 2 \pi .
\end{aligned}
$$

As seen from the eigenvalues of the anisotropy tensor in Tab. 3, the shape of the ellipses is rather similar between the different flows, where the plane jet shows the largest aspect ratio. In contrast, the principal axes of the Reynolds-stress (ansiotropy) tensor have a different inclination to the flow-aligned coordinate system, as indicated in the last column of Tab. 3 .

The inclination angle has a major influence on the transport of mean momentum normal to the principal mean-flow direction, which can be seen from the anisotropy component associated with the Reynolds-shear stress. According to the rules of coordinate transformation its value follows from the eigenvalues associated with the flow plane by

$$
b_{12}=\left(\lambda_{1}^{(b)}-\lambda_{2}^{(b)}\right) \sin \theta \cos \theta,
$$

in which $b_{12}=b_{x y}$ for plane flow and $b_{12}=b_{r z}$ for axisymmetric flow, and $\theta$ denotes the rotation angle of the principal axis system against the flow-aligned coordinate system in the flow plane.

Obviously, for given eigenvalues (or invariants, respectively), the Reynolds-shear-stress anisotropy depends on the orientation of the principal axes relative to the flow-aligned coordinate system. By definition, $b_{12}=0$ at $\theta=n \frac{\pi}{2}$ with $n=0, \pm 1, \pm 2 \ldots$, i.e. in the principal axis system, and reaches its extremes at $\theta=\frac{\pi}{4}+n \frac{\pi}{2}$ with $n=0, \pm 1, \pm 2 \ldots$, i.e. at $\theta= \pm 45^{\circ}$, associated with values

$$
b_{12}^{(e x t)}= \pm \frac{1}{2}\left(\lambda_{1}^{(b)}-\lambda_{2}^{(b)}\right)
$$

for the minimum and maximum Reynolds-shear-stress anisotropy, respectively.

Note that the sign of the shear-stress anisotropy and, hence, the sign of the inclination angle depends on the choice of the coordinate system, particularly on the orientation of the normal coordinate relative to the velocity gradient. If the normal coordinate is oriented parallel to the velocity gradient, like in boundary layers, $b_{12}<0$ and hence $\theta<0$. If it is oriented against the velocity gradient, like in jets, $b_{12}>0$ and hence $\theta>0$. For the plane mixing layer, the 
orientation has been chosen arbitrarily against the velocity gradient so that $b_{12}>0$ and $\theta>0$. Therefore, for the lateral momentum transfer, the absolute values need to be considered.

Indeed, of all flows investigated, the incompressible plane mixing layer shows an inclination angle of the principal axes of the Reynolds-stress anisotropy tensor in the constant region that is closest to the condition of maximum Reynolds-shear-stress anisotropy, particularly when referring to the data of Delville et al. [18]. This, for example, explains the difference observed between the absolute values of $b_{x y}$ in the constant region for the incompressible boundary layer and the incompressible plane mixing layer, although the eigenvalues and invariants of the Reynolds-

stress anisotropy tensor are very similar for both flows. In contrast, the difference of eigenvalues $\lambda_{1}^{(b)}-\lambda_{2}^{(b)}$ in the constant region is larger for the incompressible plane jet than for the incompressible boundary layer, which is reflected by a larger aspect ratio of the ellipsis associated with the corresponding Reynolds-stress tensor, as shown in Fig. 6 (right). However, due to the lower inclination angle of the principal axes for the incompressible plane jet, the absolute values of $b_{x y}$ for the incompressible plane jet and the incompressible boundary layer are very similar.

\section{Implications for Turbulence Modeling}

The above findings imply that, for accurately predicting turbulent free-shear flows, complete information on the Reynolds-stress anisotropy tensor, at least in the constant region, is required by the respective turbulence model. The corresponding invariants alone, however, do not account for the orientation of the principal axes of the Reynolds-stress tensor and are, therefore, insufficient for a complete description of the turbulence structure. The potential improvement will be demonstrated subsequently.

\section{V.A Eddy-Viscosity Models}

Commonly, the Reynolds-stress tensor is modeled according to the Boussinesq hypothesis,

$$
R_{i j}=-2 v_{t} S_{i j}+\frac{2}{3} k \delta_{i j}
$$

assuming, for incompressible flow, the Reynolds stresses being proportional to the strain rates

$$
S_{i j}=\frac{1}{2}\left(\frac{\partial U_{i}}{\partial x_{j}}+\frac{\partial U_{j}}{\partial x_{i}}\right)
$$

scaled by an eddy viscosity $v_{t}$. The second term in Eq. (45) corrects for the proper trace of the Reynolds-stress tensor and is omitted in models that do not provide $k$.

Under the above boundary-layer assumptions there is one dominating strain-rate component, $S_{x y}=\frac{1}{2} \frac{\partial U}{\partial y}$, so that 
the corresponding Reynolds-stress tensor simplifies to

$$
\underline{\underline{R}}=\left(\begin{array}{ccc}
\frac{2}{3} k & -v_{t} \frac{\partial U}{\partial y} & 0 \\
-v_{t} \frac{\partial U}{\partial y} & \frac{2}{3} k & 0 \\
0 & 0 & \frac{2}{3} k
\end{array}\right)
$$

Its eigenvalues

$$
\begin{aligned}
& \lambda_{1,2}^{(R)}=\frac{2}{3} k \pm\left|v_{t} \frac{\partial U}{\partial y}\right| \\
& \lambda_{3}^{(R)}=\frac{2}{3} k
\end{aligned}
$$

are associated with principal axes that are inclined by $\theta= \pm 45^{\circ}$ against the main-flow direction, depending on the sign of the velocity gradient.

Thus, for any flow governed by the incompressible boundary-layer assumptions, eddy-viscosity models will not only predict identical Reynolds-normal stresses, but also an identical orientation of the principal axes of the Reynolds-stress tensor relative to the flow-aligned coordinate system. In contrast, the above analysis of experimental data implies different non-identical Reynolds-normal stresses and different orientations of the principal axes of the Reynolds-stress tensor, depending on the particular type of flow. Note that this result is independent of the particular type of eddyviscosity model, except that the $k$-contribution might be omitted in some models.

Nevertheless, considering the Reynolds-shear stress as the most important component, eddy-viscosity models can be calibrated accordingly [78, 79]. Moreover, the models by Spalart and Allmaras [80] and Menter [81] employ the wall-distance for distinguishing boundary layers from other flows, i.e. general free-shear flows.

\section{V.B Reynolds-Stress Models}

Reynolds-stress models do not rely on the Boussinesq hypothesis (45), but involve the solution of the modeled transport equation of the Reynolds stresses, instead. The focus in Reynolds-stress modeling is on the pressure-strain correlation $\Pi_{i j}$, which is generally modeled according to the analysis of Rotta [67] as a traceless tensor. It therefore does not contribute to the budget of the specific kinetic turbulence energy $k$, but only redistributes it into the different directions, i.e. it alters the eigenvalues and principal axes of the Reynolds-stress (anisotropy) tensor. 
Table 4 Model coefficients and anisotropies

\begin{tabular}{lcccccccccc} 
Model & $C_{1}$ & $C_{1}^{*}$ & $C_{2}$ & $C_{3}$ & $C_{3}^{*}$ & $C_{4}$ & $C_{5}$ & $b_{x x}$ & $b_{y y}$ & $\left|b_{x y}\right|$ \\
\hline SSG & 3.40 & 1.8 & 4.200 & 0.8 & 1.3 & 1.250 & 0.400 & 0.201 & -0.127 & 0.160 \\
Modified & 3.72 & 0 & -0.675 & 0.8 & 0 & 1.598 & 1.424 & 0.075 & -0.083 & 0.173
\end{tabular}

For demonstration, the model of Speziale, Sarkar, and Gatski (SSG) [82] is considered, reading

$$
\begin{aligned}
\rho \Pi_{i j}= & -\left(C_{1} \rho \epsilon+\frac{1}{2} C_{1}^{*} \rho P_{k k}\right) b_{i j}+C_{2} \rho \epsilon\left(b_{i k} b_{k j}-\frac{1}{3} b_{k l} b_{k l} \delta_{i j}\right) \\
& +\left(C_{3}-C_{3}^{*} \sqrt{b_{k l} b_{k l}}\right) \rho k S_{i j} \\
& +C_{4} \rho k\left(b_{i k} S_{j k}+b_{j k} S_{i k}-\frac{2}{3} b_{k l} S_{k l} \delta_{i j}\right) \\
& +C_{5} \rho k\left(b_{i k} W_{j k}+b_{j k} W_{i k}\right)
\end{aligned}
$$

in which $S_{i j}$ denotes the strain rates according to Eq. (46) and

$$
W_{i j}=\frac{1}{2}\left(\frac{\partial U_{i}}{\partial x_{j}}-\frac{\partial U_{j}}{\partial x_{i}}\right)
$$

are the rotation rates. The model coefficients are given in Tab. 4 together with the associated anisotropies according to the equilibrium condition [13), assuming isotropic dissipation [72]. The anisotropies do not agree with any of the data sets in Tab. 2, except for $b_{x y}$ being close to the plane-mixing layer value in the fourth row.

In order to demonstrate potential improvement, the model has been recalibrated to the full set of Reynolds-stress anisotropies for the constant region of the plane mixing layer according to the data of Delville et al. [18] in Tab. 2. For this purpose, the modeled pressure-strain correlation (50) is inserted into the turbulent equilibrium condition (13). Exploiting the boundary-layer simplifications, a system of equations is obtained according to the individual tensor components, relating the model coefficients to the anisotropies in the constant region. The set of recalibrated coefficients is termed "Modified" and is also included in Tab. 4. This set of coefficients has been implemented into DLR's flow solver TAU with the SSG-part of the SSG/LRR- $\omega$ model [83-85], which is active outside boundary layers and, thus, in free shear-layer flows developing at a distance from solid walls.

Computations have been carried out for the plane mixing layer, specifying inflow conditions according to the experiment by Delville et al. [18] as provided on the Turbulence Modeling Resource (TMR) website [55]. The computations have been carried out on the three finest structured grids provided on the TMR website [55] with $N=42,176$ cells, $N=168,704$ cells and $N=674,816$ cells, respectively. The results confirmed that the second finest grid is sufficient for obtaining a grid independent solution. Profile data have been extracted from the simulations 
at $x=950 \mathrm{~mm}$ downstream of the trailing edge, which is the most downstream position where experimental Reynolds stresses are provided.

Figure 7 shows the comparison of the predicted mean-velocity and Reynolds-stress profiles with the experimental data by Delville et al. [18]. While there is almost no influence of the respective set of coefficients on the mean-velocity profile, significant differences are observed for the Reynolds-stress profiles. With the SSG-coefficients, the peak Reynolds stresses are generally missed, whereas, with the modified coefficients, good agreement with the experimental data is achieved in the center of the mixing layer, except for $R_{z z}$ that nevertheless is improved. This confirms that the pressure-strain correlation controls the eigensystem of the Reynolds-stress tensor and underlines the importance of a proper calibration for the Reynolds-stress anisotropies in the constant region. Nevertheless, note also the deviations towards the edges of the plane mixing layer, indicating the limits of the underlying assumptions. While certainly valid in the center of the shear layer, towards its edges, the flow requires a different treatment, probably due to the presence of large coherent structures.

The above procedure demonstrates exemplarily how certain model characteristics can be controlled by a rational choice of the constant closure coefficients. It is a proof of concept, not a validation of an improved model. Note that the methodology is supposed to allow addressing also the round jet/plane jet anomaly, which is nevertheless deferred to the comprehensive development of an improved model.

\section{V.C Modeling Implications}

The results obtained for the incompressible mixing layer reveal the potential of improving turbulence models, particularly differential Reynolds stress models, by proper calibration of the individual set of anisotropies in the constant region of incompressible turbulent shear layers. Since these values vary between different shear layer flows, an improved model would need to automatically adjust to the most suitable set of coefficients as implied in Ref. [86]. Particularly, for its applicability in an industrial environment, this would require an automatic identification of the local flow type. Even though it is supposed to be difficult, the development of such a self-adaptive model might be rewarding and has therefore been recently initiated.

\section{Conclusions}

It has been theoretically shown that, if a flow is governed by the incompressible boundary-layer equations and there is an equilibrium between production, dissipation and the pressure-strain correlation, the self-similarity of the mean velocity and the specific Reynolds-shear stress implies that the Reynolds-stress anisotropies are constant. The analysis of experimental data for the incompressible plane jet, the incompressible axisymmetric jet and the incompressible plane mixing layer confirms the existence of such a layer with approximately constant Reynolds-stress anisotropies.

The values of the Reynolds-stress anisotropies obtained in the constant region differ between the flows under 
investigation. Evaluation of the corresponding invariants, eigenvalues and principal axes shows that, even if the invariants and eigenvalues are similar, the principal axes are inclined differently against the flow-aligned coordinate system, giving rise to different Reynolds-shear stress anisotropy.

Eddy-viscosity models always predict the principal axes of the Reynolds-stress tensor to be inclined by $\theta= \pm 45^{\circ}$ to the main-flow direction of an incompressible boundary or free-shear layer. In contrast, with Reynolds-stress models the predicted Reynolds-stress anisotropy tensor and, hence, its eigensystem are controlled by the pressure-strain correlation. Potential improvement has been demonstrated for the plane mixing layer by recalibrating the SSG-model according to the Reynolds-stress anisotropies deduced from experimental data.

It is conjectured that observed model failures, like the round-jet/plane-jet anomaly or the under-estimation of Reynolds-shear stress levels in separated shear layers, might be associated with a model calibration that is unsuitable for the respective flow situation. In this case, improvement might be achieved by a self-adaptive model, switching automatically between the respective sets of tailored closure coefficients, depending on the local flow type.

\section{Acknowledgements}

Inspiring discussions with Dr. T. Knopp at DLR Göttingen are gratefully acknowledged.

\section{References}

[1] Bush, B., Chyczewski, T., Duraisamy, K., Eisfeld, B., Rumsey, C., and Smith, B., "Recommendations for Future Efforts in RANS Modeling and Simulation," AIAA Paper 2019-0317, 2019.

https://doi.org/10.2514/6.2019-0317

[2] Wilcox, D. C., “Turbulence Modeling for CFD,” DCW Industries, 3rd Edition, 2006.

[3] Brown, G. L., Roshko, A., "On density effects and large structures in turbulent mixing layers," Journal of Fluid Mechanics, Vol. 64, 1974, pp. 775-816.

https://doi.org/10.1017/S002211207400190X

[4] Roshko, A., "Structure of Turbulent Shear Flows: A New Look," AIAA Journal, Vol. 14, No. 10, 1976, pp. $1349-1357$. https://doi.org/10.2514/3.61477

[5] Görtler, H., "Berechnung von Aufgaben der freien Turbulenz auf Grund eines neuen Näherungsansatzes," Zeitschrift für Angewandte Mathematik und Mechanik, Vol. 22, 1942, pp. 244-254.

https://doi.org/10.1002/zamm.19420220503

[6] Prandtl, L., "Bemerkungen zur Theorie der freien Turbulenz," Zeitschrift für Angewandte Mathematik und Mechanik, Vol. 22, 1942, pp. 241-243.

https://doi.org/10.1002/zamm.19420220502 
[7] Förthmann, E., “Über turbulente Strahlausbreitung,” Ingenieurarchiv, Vol. 5, 1934, pp. 42-54.

https://doi.org/10.1007/BF02086177

[8] Corrsin, S., "Investigation of Flow in an Axially Symmetrical Heated Jet of Air," NACA Wartime Report W-94, 1943.

[9] Liepmann, H. W., Laufer, J., “Investigations of Free Turbulent Mixing,” NACA Technical Note 1257, 1947.

[10] Bradbury, L. J. S., “The structure of a self-preserving turbulent plane jet,” Journal of Fluid Mechanics, Vol. 23, 1965, pp. 31-64.

https://doi.org/10.1017/S0022112065001222

[11] Heskestad, G., "Hot-Wire Measurements in a Plane Turbulent Jet," Journal of Applied Mechanics, Vol. 32, 1965 , pp. $721-734$. https://doi.org/10.1115/1.3627310

[12] Gutmark, E., Wygnanski, I., “The planar turbulent jet,” Journal of Fluid Mechanics, Vol. 73, 1976, pp. 465-495. https://doi.org/10.1017/S0022112076001468

[13] Antonia, R. A., Bilger, R. W., “An experimental investigation of an axisymmetric jet in a co-flowing air stream,” Journal of Fluid Mechanics, Vol. 61, 1973, pp. 805-822. https://doi.org/10.1017/S0022112073000959

[14] Wygnanski, I., Fiedler, H., "Some measurements in the self-preserving jet," Journal of Fluid Mechanics, Vol. 38, 1969, pp. $577-612$. https://doi.org/10.1017/S0022112069000358

[15] Hussein, H. J., Capp, S. P., George, W. K., "Velocity measurements in a high-Reynolds-number, momentum-conserving, axisymmetric, turbulent jet," Journal of Fluid Mechanics, Vol. 258, 1994, pp. 31-75.

https://doi.org/10.1017/S002211209400323X

[16] Wygnanski, I., Fiedler, H. E., “The two-dimensional mixing region,” Journal of Fluid Mechanics, Vol. 41, 1970, $327-361$. https://doi.org/10.1017/S0022112070000630

[17] Tavoularis, S., Corrsin, D., “The structure of a turbulent shear layer embedded in turbulence," Physics of Fluids, Vol. 30, 1987, pp. 3025-3033.

https://doi.org/10.1063/1.866081

[18] Delville, J., Bellin, S., Garm, J. H., Bonnet, J. P., "Analysis of Structures in a Turbulent, Plane Mixing Layer by Use of a Pseudo Flow Visualization Method Based on Hot-Wire Anemometry.” In: Fernholz, H. H., Fiedler, H. E. (Eds.), “Advances in Turbulence 2," Springer, 1989, pp. 251-256.

[19] Bell, J. H., Mehta, R. D., “Development of a Two-Stream Mixing Layer from Tripped and Untripped Boundary Layers,” AIAA Journal, Vol. 28 No. 12, 1990, pp. 2034-2042.

https://doi.org/10.2514/3.10519 
[20] Mehta, R. D., "Effect of velocity ratio on plane mixing layer development: Influence of the splitter plate wake," Experiments in Fluids, Vol. 10, 1991, pp. 194-204.

https://doi.org/10.1007/BF00190389

[21] Benocci, C., Tavoularis, S., Bonnet, J.-P., Leuchter, O., Rodi, W., Onorato, M., van der Vegt, J J. W., Jimenez, J., Smith, P. D., Moser, R, S., Purtell, L. P., "A Selection of Test Cases for the Validation of Large-Eddy Simulations of Turbulent Flow R,” AGARD-Report AGARD-AR-345, 1998.

[22] University of Madrid, Turbulent Database, AGARD, https://torroja.dmt.upm.es/turbdata/, retrieved 20 July 2020.

[23] Ball, C.G., Fellouah, H., Pollard, A. "The flow field in turbulent round free jets," Progress in Aerospace Sciences, Vol. 50, 2012, pp. 1-26.

https://doi.org/10.1016/j.paerosci.2011.10.002

[24] Ferdman, E., Ötügen, M.V., Kim, S., "Effect of Initial Velocity Profile on the Development of Round Jets," Journal of Propulsion and Power, Vol. 16 No. 4, 2000, pp. 676-686.

https://doi.org/10.2514/2.5627

[25] Antonia, R.A., Zhao, Q., “Effect of initial conditions on a circular jet,” Experiments in Fluids, Vol. 31, 2001 , pp. $319-323$. https://doi.org/10.1007/s003480100289

[26] Mi, J., Nobes, D.S., Nathan, G.J., "Influence of jet exit conditions on the passive scalar field of an axisymmetric free jet," Journal of Fluid Mechanics, Vol. 432, 2001, pp. 91-125.

https://doi.org/10.1017/S0022112000003384

[27] Romano, G.P., Antonia, R.A., "Longitudinal and transverse structure functions in a turbulent round jet: effect of initial conditions and Reynolds number," Journal of Fluid Mechanics, Vol. 436, 2001, pp.231-248.

https://doi.org/10.1017/S0022112001003901

[28] Xu, G., Antonia, R.A., “Effect of different initial conditions on a turbulent round free jet,” Experiments in Fluids, Vol. 33, 2002, pp. 677-683.

https://doi.org/10.1007/s00348-002-0523-7

[29] Kwon, S.J., Seo, I.W., "Reynolds number effects on the behavior of a non-buoyant round jet," Experiments in Fluids, Vol. 38, 2005, pp. 801-812.

https://doi.org/10.1007/s00348-005-0976-6

[30] Iqbal, M.O., Thomas, F.O., "Coherent structure in a turbulent jet via a vector implementation of the proper orthogonal decomposition,” Journal of Fluid Mechanics, Vol. 571, 2007, pp. 281-326.

https://doi.org/10.1017/S0022112006003351 
[31] Weisgraber, T.H., Liepmann, D., "Turbulent structure during transition to self-similarity in a round jet," Experiments in Fluids, Vol. 24, 1998, pp. 210-224.

https://doi.org/10.1007/s003480050168

[32] Gamard, S., George, W.K., Jung, D., Woodward, S., "Application of a "slice" proper orthogonal decomposition to the far field of an axisymmetric turbulent jet," Physics of Fluids, Vol. 14 No. 7, 2002, pp. 2515-2522.

https://doi.org/10.1063/1.1471875

[33] Gamard, S., Jung, D., George, W.K., "Downstream evolution of the most energetic modes in a turbulent axisymmetric jet at high Reynolds number. Part 2. The far-field region,” Journal of Fluid Mechanics, Vol. 514, 2004, pp. 205-230.

https://doi.org/10.1017/S0022112004000175

[34] Shineeb, A.-M., Balachandar, R., Bugg, J.D., "Analysis of Coherent Structures in the Far-Field Region of an Axisymmetric Free Jet Identified Using Particle Image Velocimetry and Proper Orthogonal Decomposition,” Journal of Fluids Engineering, Vol. 130, 2008, 011202.

https://doi.org/10.1115/1.2813137

[35] Agrawal, A., Prasad, A.K., "Properties of vortices in the self-similar turbulent jet," Experiments in Fluids, Vol. 33, 2002, 565-577.

https://doi.org/10.1007/s00348-002-0507-7

[36] Agrawal, A., Prasad, A.K., "Organizational Modes of Large-Scale Vortices in an Axisymmetric Turbulent Jet," Flow, Turbulence and Combustion, Vol. 68, 2002, 359-377.

https://doi.org/10.1023/A:1021711013263

[37] Ewing, D., Frohnapfel, B., George, W.K., Pedersen, J.M., Westerweel, J., “Two-point similarity in the round jet," Journal of Fluid Mechanics, Vol. 577, 2007, pp. 309-330.

https://doi.org/10.1017/S0022112006004538

[38] Burattini, P., Antonia, R.A., Danaila, L., "Similarity in the far field of a turbulent round jet," Physics of Fluids, Vol. 17, 2005, 025101.

https://doi.org/10.1063/1.1833414

[39] Deo, R.C., Nathan, G.J., Mi, J., "Similarity analysis of the momentum field of a subsonic, plane air jet with varying jet-exit and local Reynolds numbers," Physics of Fluids, Vol. 25, 2013, 015115.

https://doi.org/10.1063/1.4776782

[40] Paillat, S., Kaminski, E., "Second-order model of entrainment in planar turbulent jets at low Reynolds number," Physics of Fluids, Vol. 26, 2014, 045110.

https://doi.org/10.1063/1.4871521 
[41] Deo, R. C., Mi, J., Nathan, G. J., "The influence of nozzle-exit geomeric profile on statistical properties of a turbulent plane jet," Experimental Thermal and Fluid Science, Vol. 32, 2007, 545-559.

https://doi.org/10.1016/j.expthermflusci.2007.06.004

[42] Deo, R. C., Mi, J., Nathan, G. J., “The influence of Reynolds number on a plane jet,” Physics of Fluids, Vol. 20, $2008,075108$. https://doi.org/10.1063/1.2959171

[43] Gordeyev, S. V., Thomas, F. O., "Coherent structure in the turbulent planar jet. Part 1. Extraction of proper orthogonal decomposition eigenmodes and their self-similarity," Journal of Fluid Mechanics, Vol. 414, 2000, 145-194.

https://doi.org/10.1017/S002211200000848X

[44] Matsubara, M., Alfredsson, P.H., Segalini, A., "Linear modes in a planar turbulent jet," Journal of Fluid Mechanics, Vol. 888, 2020, A26.

https://doi.org/10.1017/jfm.2020.25

[45] Cafiero, G., Vassilicos, J. C., “Non-equilibrium turbulence scalings and self-similarity in turbulent planar jets," Proceedings of the Royal Society A, Vol. 475, 2019, 20190038.

https://doi.org/10.1098/rspa.2019.0038

[46] Naka, Y., Omori, T., Obi, S., Masuda, S., "Simultaneous measurements of fluctuating velocity and pressure in a turbulent mixing layer," International Journal of Heat and Fluid Flow, Vol. 18, 2006, 737-746.

https://doi.org/10.1016/j.ijheatfluidflow.2006.02.023

[47] Li, C.-T-, Chang, K.-C., Wang, M.-R., "PIV measurements of turbulent flow in planar mixing layer," Experimental Thermal and Fluid Science, Vol. 33, 2009, 527-537.

https://doi.org/10.1016/j.expthermflusci.2008.11.006

[48] Guo, F., Chen, B., Guo, L., Zhang, X., "Effects of Velocity Ratio on turbulent Mixing Layer at high Reynolds number,” Journal of Physics: Conference Series, Vol. 147, 2009, 012049.

https://doi.org/10.1088/1742-6596/147/1/012049

[49] Buxton, O. R. H., Ganapathisubramani, B., "PIV measurements of convection velocities in a turbulent mixing layer," Journal of Physics: Conference Series, Vol. 318, 2011, 052038.

https://doi.org/10.1088/1742-6596/318/5/052038

[50] Buxton, O. R. H., de Kat, R., Ganapathisubramani, B., "The convection of large and intermediate scale fluctuations in a turbulent mixing layer," Physics of Fluids, Vol. 25, 2013, 125105.

https://doi.org/10.1063/1.4837555

[51] Buxton, O. R. H., Ganapathisubramani, B., "Concurrent scale interactions in the far-field of a turbulent mixing layer," Physics of Fluids, Vol. 26, 2014, 125106.

https://doi.org/10.1063/1.4903970 
[52] Loucks, R. B., Wallace, J. M., "Velocity and velocity gradient based properties of a turbulent plane mixing layer," Journal of Fluid Mechanics, Vol. 699, 2012, 280-319.

https://doi.org/10.1017/jfm.2012.103

[53] Li, N., Balaras, E., Wallace, J. M., "Passive Scalar Transport in a Turbulent Mixing Layer," Flow, Turbulence and Combustion, Vol. 85, 2010, 1-24.

https://doi.org/10.1007/s10494-010-9249-4

[54] The University of Manchester, "European Research Community on Flow, Turbulence and Combustion Database," http://cfd.mace.manchester.ac.uk/ercoftac/, retrieved 20 July 2020.

[55] Rumsey, C. L., “Turbulence Modeling Resource,” https://turbmodels.larc.nasa.gov, retrieved 20 July 2020.

[56] Delville, J., Ukeiley, L., Cordier, L., Bonnet, J. P., Glauser, M., "Examination of large-scale structures in a turbulent plane mixing layer. Part 1. Proper orthogonal decomposition,” Journal of Fluid Mechanics, Vol. 391, 1999, 91-122. https://doi.org/10.1017/S0022112099005200

[57] Druault, P., Delville, J., Bonnet, J.-P., "Experimental 3D Analysis of the Large Scale Behaviour of a Plane Turbulent Mixing Layer," Flow, Turbulence and Combustion, Vol. 74, 2005, 207-233.

https://doi.org/10.1007/s10494-005-4136-0

[58] Dimotakis, P. E., “The mixing transition in turbulent flows,” Journal of Fluid Mechanics, Vol. 409, 2000, 69-98. https://doi.org/10.1017/S0022112099007946

[59] Uddin, M, Pollard, A., "Self-similarity of coflowing jets: The virtual origin,” Physics of Fluids, Vol. 19, $2007,068103$. https://doi.org/10.1063/1.2740709

[60] Shin, D., Sandberg, R. D., Richardson, E. S., "Self-similarity of fluid residence time statistics in a turbulent round jet," Journal of Fluid Mechanics, Vol. 823. 2017, 1-25.

https://doi.org/10.1017/jfm.2017.304

[61] Schlichting, H., “Laminare Strahlausbreitung,", Zeitschrift für Angewandte Mathematik und Mechanik, Vol. 13, 1933, $260-263$. https://doi.org/10.1002/zamm.19330130403

[62] Pope, S. B., “Turbulent Flows,” Cambridge University Press, 2000.

[63] Lumley, J. L., “Computational Modeling of Turbulent Flows,” Advances in Applied Mechanics, Vol. 18, 1979, pp. 123-176. https://doi.org/10.1016/S0065-2156(08)70266-7

[64] Hinze, J. O., “Turbulence,” McGraw-Hill, 2nd Edition, 1975.

[65] Rotta, J. C., “Turbulente Strömungen,” Teubner, 1972. 
[66] Launder, B. E., Reece, G. J., Rodi, W., "Progress in the Development of a Reynolds-Stress Turbulence Closure," Journal of Fluid Mechanics, Vol. 68, 1975, pp. 537-566.

https://doi.org/10.1017/S0022112075001814

[67] Rotta, J., "Statistische Theorie nichthomogener Turbulenz," Zeitschrift für Physik, Vol. 129, 1951, pp. 547-572. https://doi.org/10.1007/BF01329645

[68] Corrsin, S., "Local isotropy in turbulent shear flow," NACA Research Memorandum RM 58B11, 1958.

[69] Saddoughi, S. G., Veeravalli, S. V., "Local isotropy in turbulent boundary layers at high Reynolds number," Journal of Fluid Mechanics, Vol. 268, 1994, pp. 333-372.

https://doi.org/10.1017/S0022112094001370

[70] George, W. K., “The Self-Preservation of Turbulent Flows and Its Relation to Initial Conditions and Coherent Structures.” In: George, W. K., Arndt, R. (Eds.), “Advances in Turbulence,” Springer, 1989, pp. 39-73.

[71] Durbin, P. A., Pettersson Reif, B. A., “Statistical Theory and Modeling for Turbulent Flows,” Wiley, Reprint 2003.

[72] Abid, R., Speziale, C. G., "Predicting equilibrium states with Reynolds stress closures in channel flow and homogeneous shear flow," Physics of Fluids A, Vol. 5, 1993, pp. 1776-1782.

https://doi.org/10.1063/1.858852

[73] Bradshaw, P., Ferriss, D. H., Atwell, N. P., "Calculation of boundary-layer development using the turbulent energy equation," Journal of Fluid Mechanics, Vol. 28, 1967, pp. 593-616.

https://doi.org/10.1017/S0022112067002319

[74] Dairay, T., Obligado, M., Vassilicos, J. J., “Non-equilibrium scaling laws in axisymmetric turbulent wakes,” Journal of Fluid Mechanics, Vol. 781, 2015, pp. 166-195.

https://doi.org/10.1017/jfm.2015.493

[75] Girimaij, S. S., Jeong, E., Poroseva, S. V., "Pressure-strain correlation in homogeneous anisotropic turbulence subject to rapid strain-dominated distortion," Physics of Fluids, Vol. 15, 2003, 3209-3222.

https://doi.org/10.1063/1.1608012

[76] Eisfeld, B., "Reynolds Stress Anisotropy in Self-Preserving Turbulent Shear Flows," Internal Report, DLR-IB-AS-BS-2017$106,2017$.

[77] Kim, K. U., Elliott, G. S., Dutton, J. C., “Three-Dimensional Experimental Study of Compressible Effects on Turbulent Free Shear Layers," AIAA Journal, Vol. 58, No. 1, 2020, pp. 133-147.

https://doi.org/10.2514/1.J058556

[78] Poroseva, S. V., Bézard, H., “On Ability of Standard k- $\epsilon$ Model to Simulate Aerodynamic Turbulent Flows,” Computational Fluid Dynamics Journal, Special Issue, 2001, 627-623. 
[79] Poroseva, S., Iaccarino, G., "Simulating separated flows using the $k-\epsilon$ model," Center for Turbulence Research, Annual Research Briefs, 2001, 375-384.

[80] Spalart, P. R. and Allmaras, S. R., “One-Equation Turbulence Model for Aerodynamic Flows,” Recherche Aèrospatiale, Vol. 1, 1994, pp. 5-21.

[81] Menter, F. R., “Two-Equation Eddy-Viscosity Turbulence Models for Engineering Applications,” AIAA Journal, Vol. 32, No. 8, 1994, pp. 1598-1605.

https://doi.org/10.2514/3.12149

[82] Speziale, C. G., Sarkar, S., Gatski, T. B., "Modelling the pressure-strain correlation of turbulence: an invariant dynamical systems approach,” Journal of Fluid Mechanics, Vol. 227, 1991. pp- 245-272.

https://doi.org/10.1017/S0022112091000101

[83] Eisfeld, B., Brodersen, O., “Advanced Turbulence Modelling and Stress Analysis for the DLR-F6 Configuration,” AIAA Paper 2005-4727, 2005.

https://doi.org/10.2514/6.2005-4727

[84] Cécora, R.-D., Radespiel, R., Eisfeld, B., Probst, A., "Differential Reynolds-Stress Modeling for Aeronautics," AIAA Journal, Vol. 53 No. 3, 2015, pp. 739-755.

https://doi.org/10.2514/1.J053250

[85] Eisfeld, B., Rumsey, C., Togiti, V., "Verification and Validation of a Second-Moment Closure Model," AIAA Journal, Vol. 54 No. 5, 2016, pp. 1524-1541.

https://doi.org/10.2514/1.J054718

[86] Poroseva, S. V., "Modeling the "rapid" part of the velocity/pressure-gradient correlation in inhomogeneous turbulence," Center for Turbulence Research, Annual Research Briefs, 2001, 367-374. 

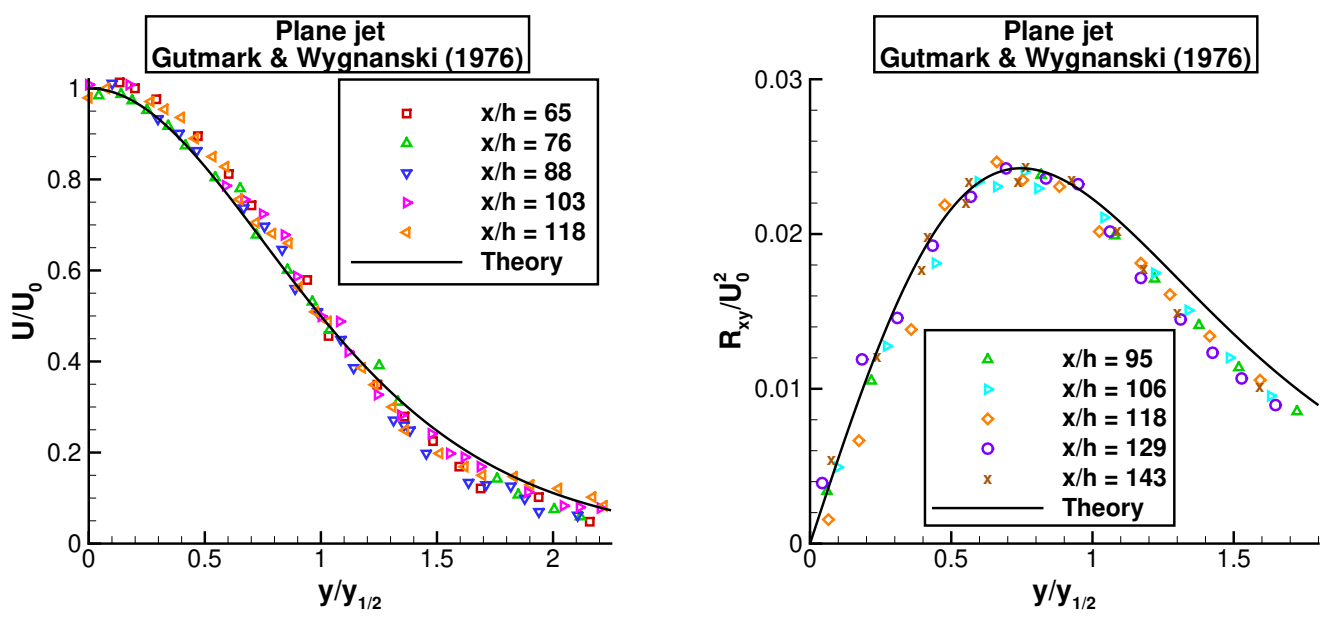

(a) Incompressible plane jet, experiment of Gutmark and Wygnanski [12].
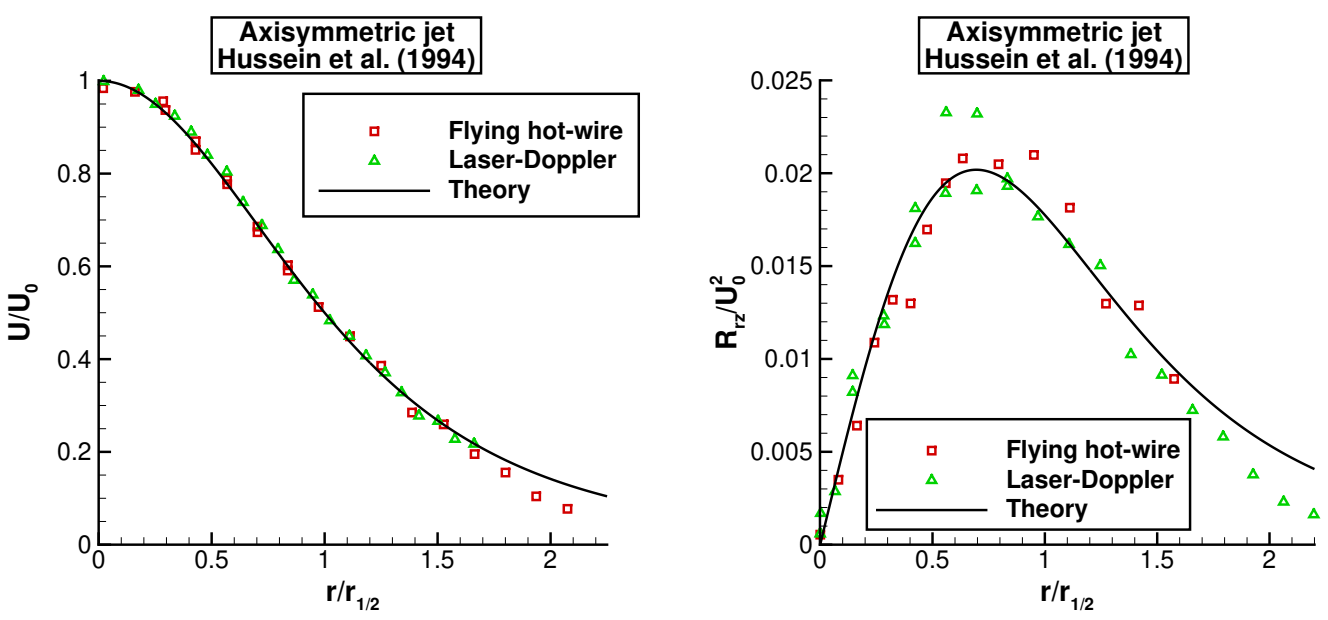

(b) Incompressible axisymmetric jet, experiment of Hussein et al. [15].
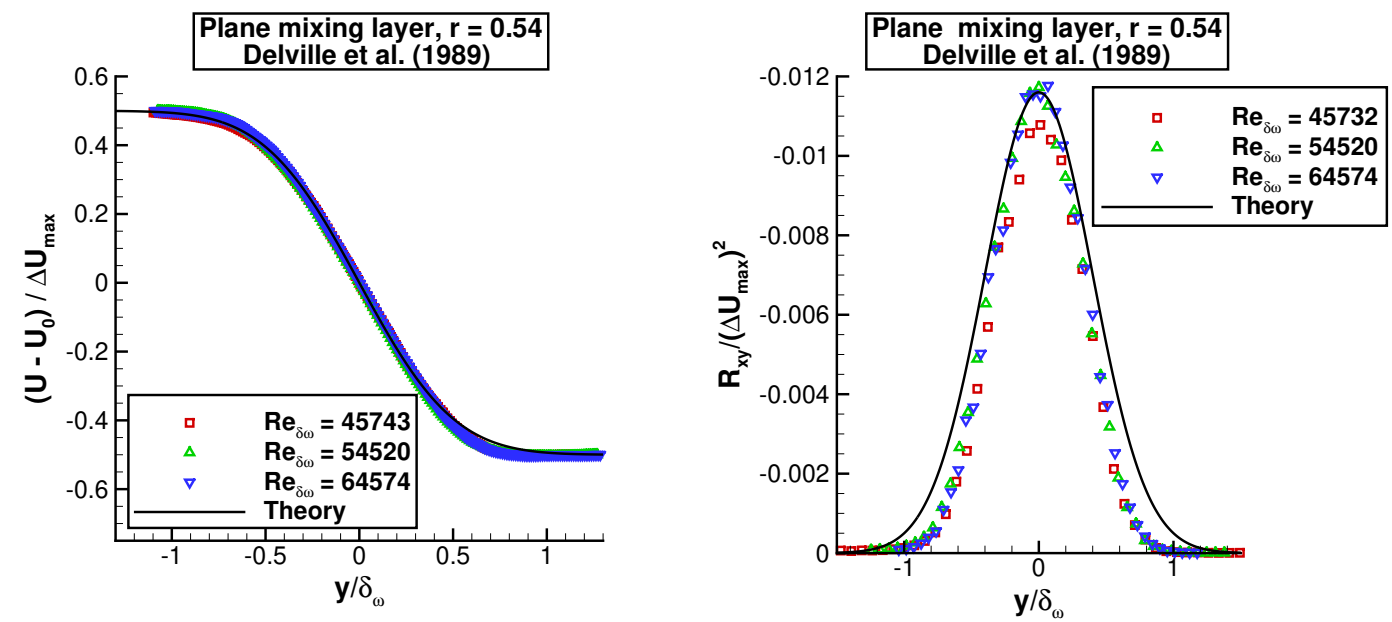

(c) Incompressible plane mixing layer, experiment of Delville et al. [18] at velocity ratio $r=0.54$.

Fig. 1 Profiles of the non-dimensional mean velocity (left) and Reynolds-shear stress (right). Comparison of experimental data with self-similar solution by Görtler [5]. 


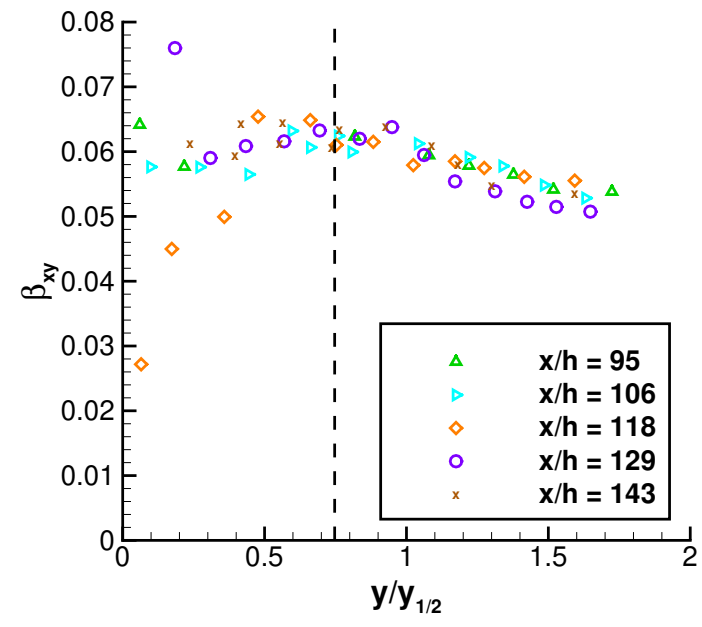

(a) Reynolds-shear stress.

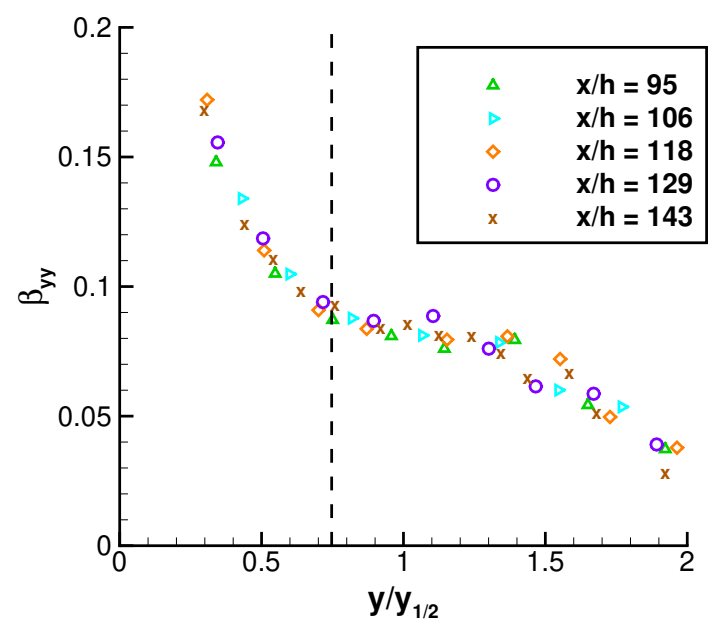

(c) Reynolds-normal stress, normal direction.

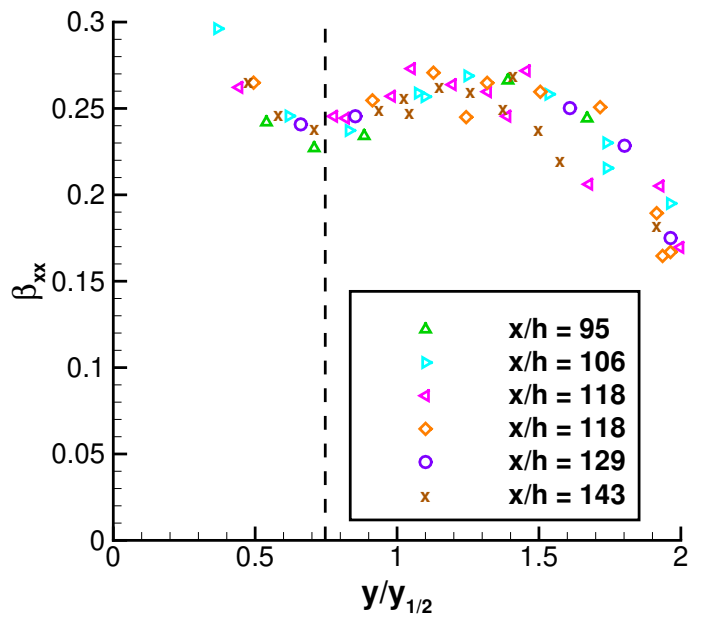

(b) Reynolds-normal stress, streamwise direction.

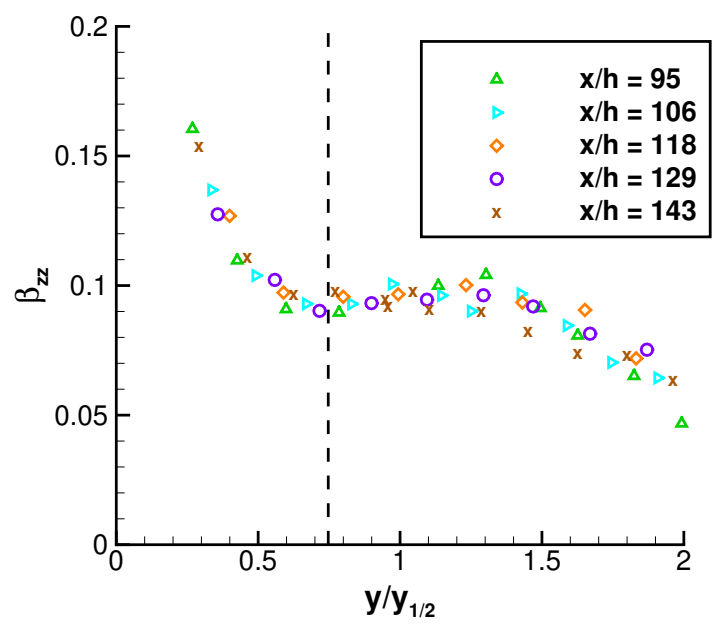

(d) Reynolds-normal stress, spanwise direction.

Fig. 2 Reynolds-stress coefficients $\beta_{i j}$ for the incompressible plane jet, experiment of Gutmark and Wygnanski [12]. $x / h=$ distance from virtual origin in terms of orifice width $h$. Dashed line indicates theoretical position of maximum specific Reynolds-shear stress. 


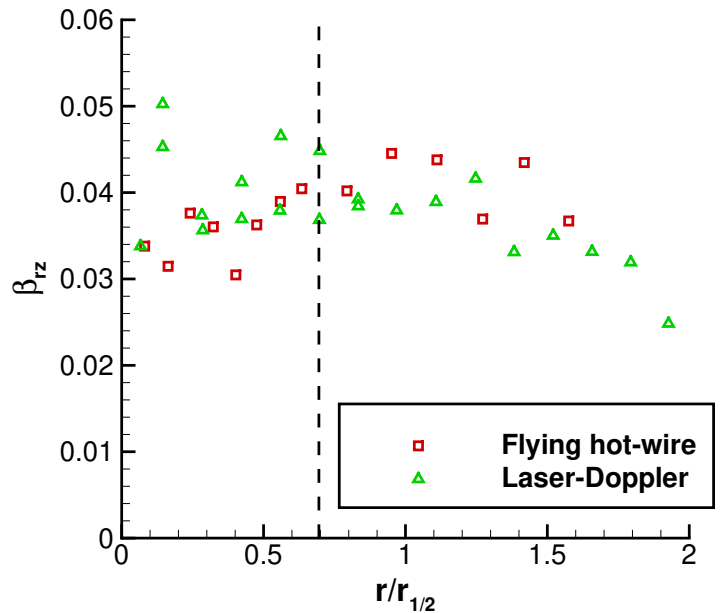

(a) Reynolds-shear stress.

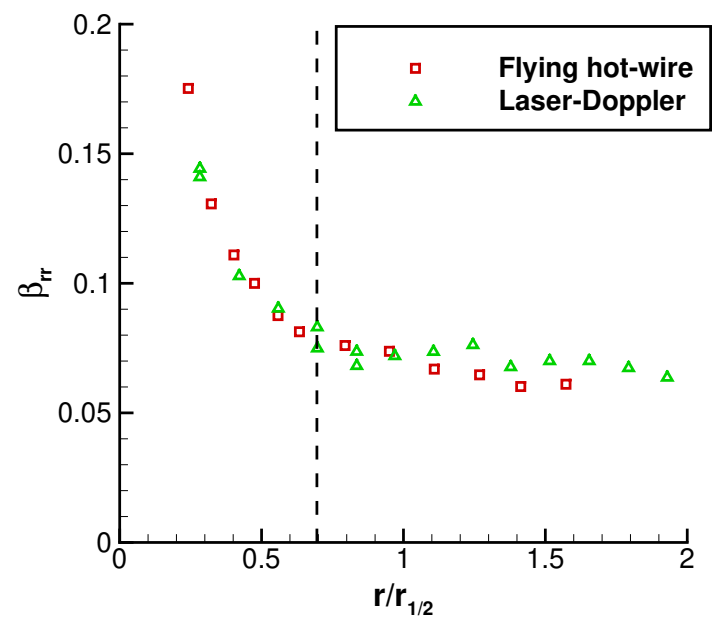

(c) Reynolds-normal stress, radial direction.

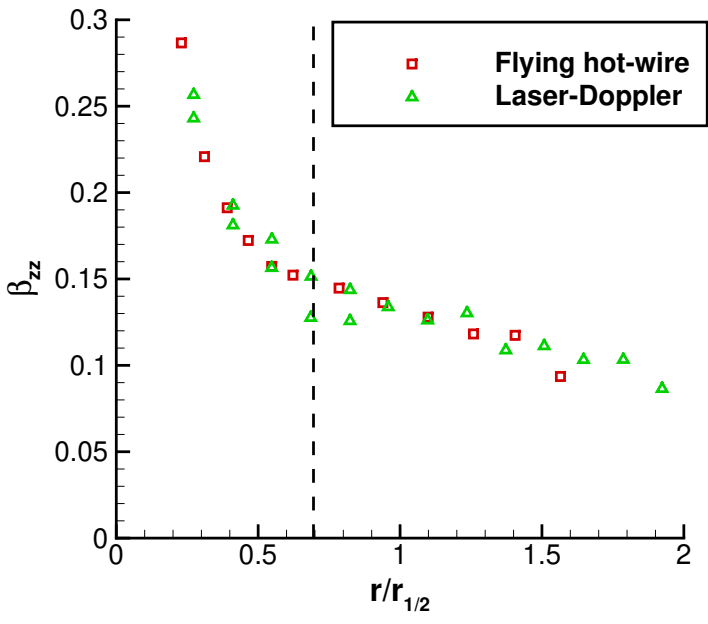

(b) Reynolds-normal stress, axial direction.

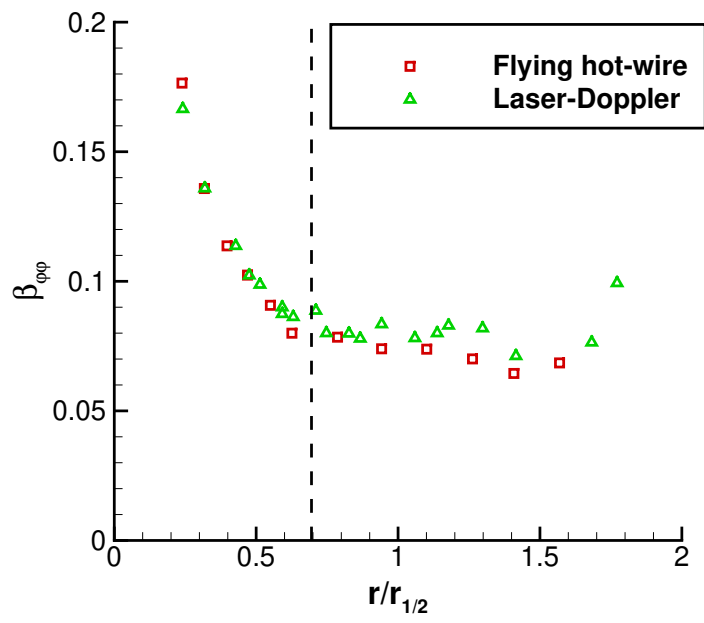

(d) Reynolds-normal stress, tangential direction.

Fig. 3 Reynolds-stress coefficients $\beta_{i j}$ for the incompressible axisymmetric jet, experiment of Hussein et al [15]]. Dashed line indicates theoretical position of maximum specific Reynolds-shear stress. 


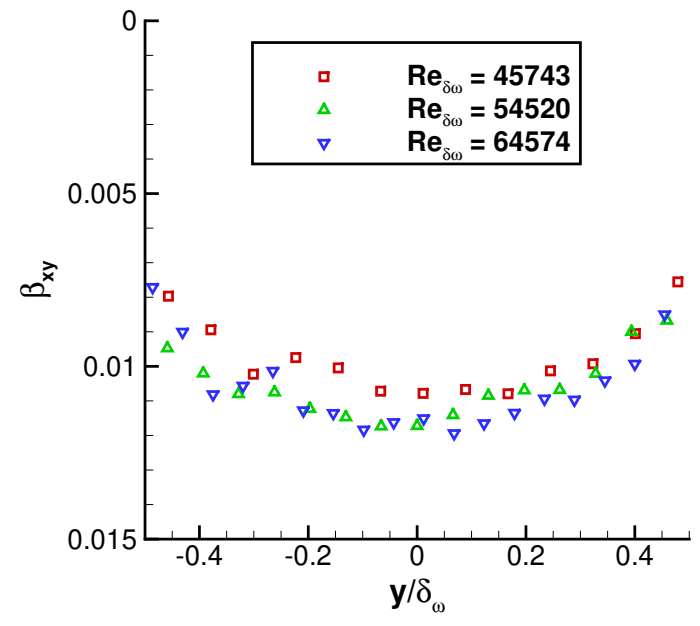

(a) Reynolds-shear stress.

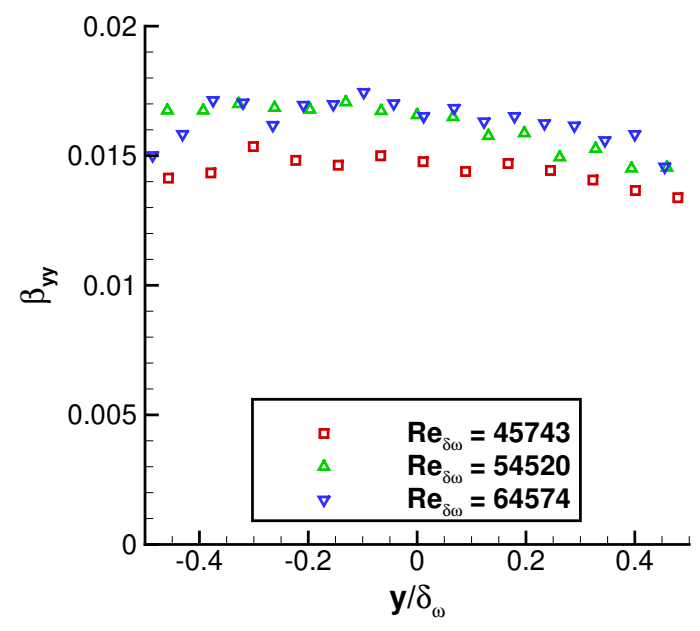

(c) Reynolds-normal stress, normal direction.

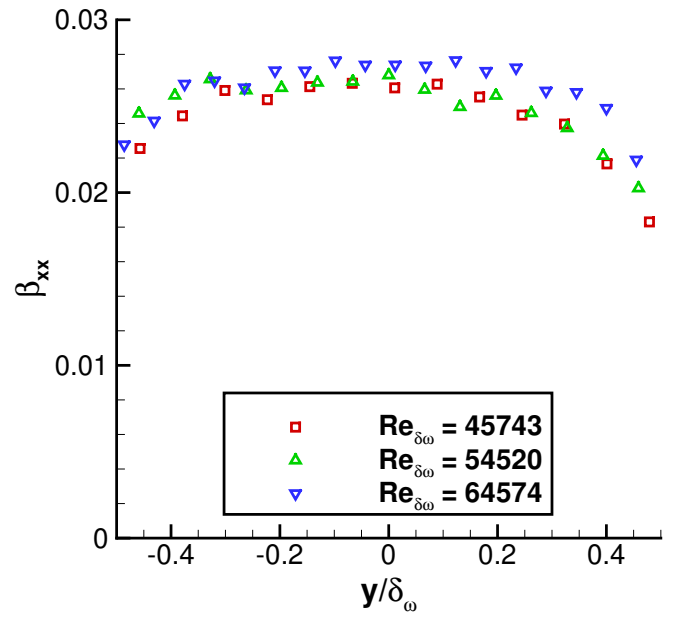

(b) Reynolds-normal stress, streamwise direction.

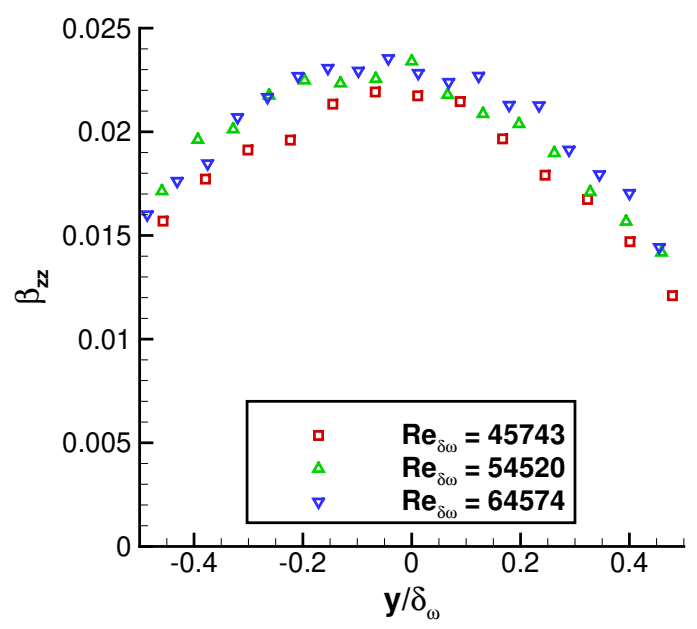

(d) Reynolds-normal stress, spanwise direction.

Fig. 4 Reynolds-stress coefficients $\beta_{i j}$ for the incompressible plane mixing layer, experiment of Delville et al. [18] at $r=0.54$. $\operatorname{Re}_{\delta_{\omega}}=\Delta U_{\max } \delta_{\omega} / v=$ local Reynolds number. 

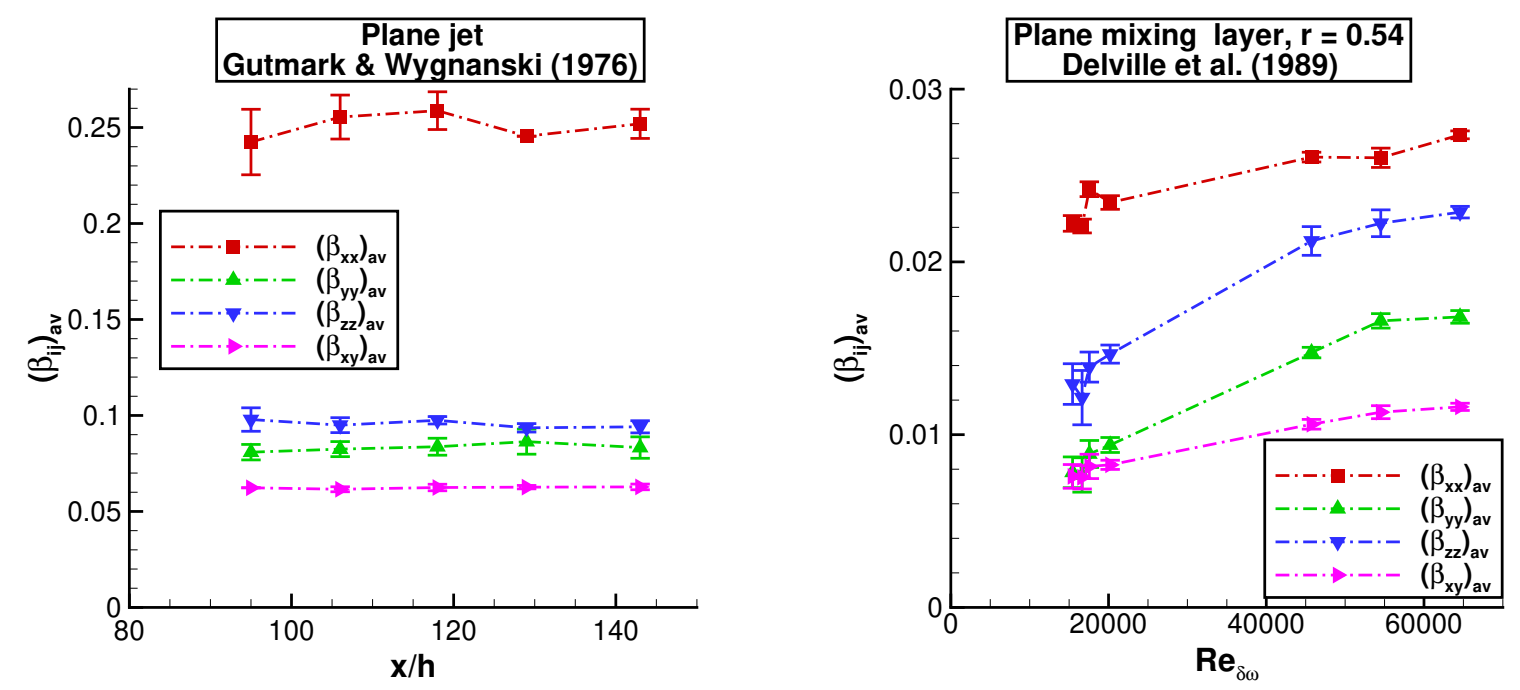

(a) Incompressible plane jet, experiment of Gutmark and Wygnanski $\quad$ (b) Incompressible plane mixing layer, experiment of Delville et al. [18] [12]. $x / h=$ distance from virtual origin in terms of nozzle width $h$. at $r=0.54$. $R e_{\delta_{\omega}}=\Delta U_{\max } \delta_{\omega} / v=$ local Reynolds number.

Fig. 5 Averaged Reynolds-stress coefficients $\left(\beta_{i j}\right)_{a v}$. Error bars indicate standard deviation of averaging.

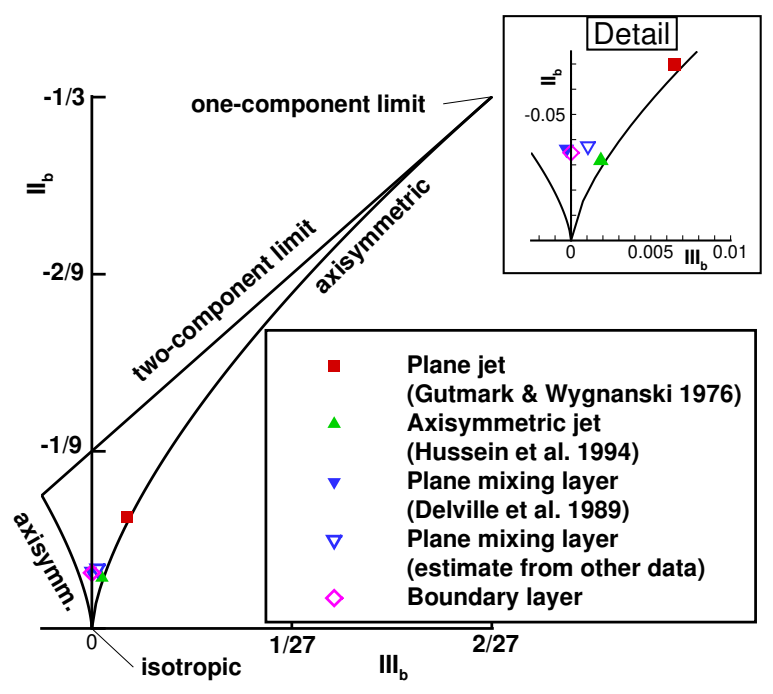

(a) Invariant map.

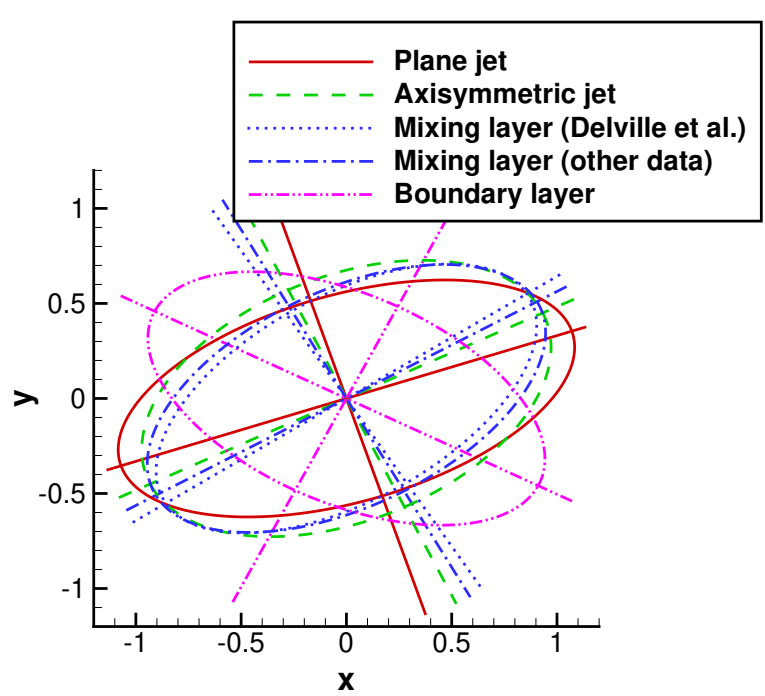

(b) Eigensystems

Fig. 6 Invariant map and eigensystems of the Reynolds-stress anisotropy tensor. 


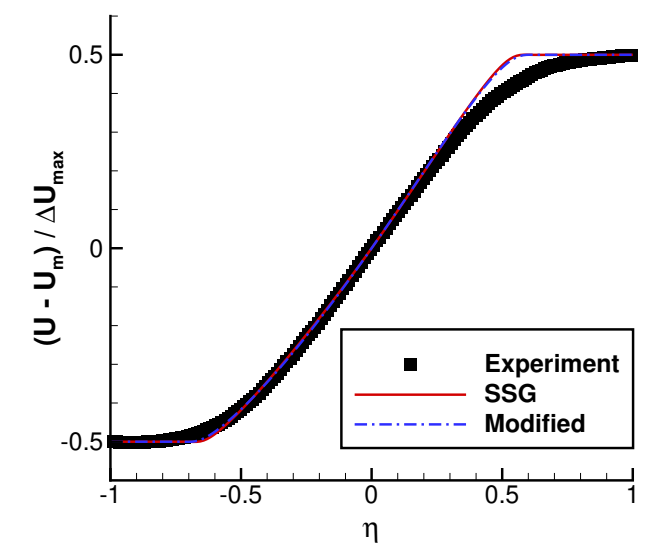

(a) Mean velocity profile. $U_{m}=\frac{1}{2}\left(U_{\min }+U_{\max }\right)$

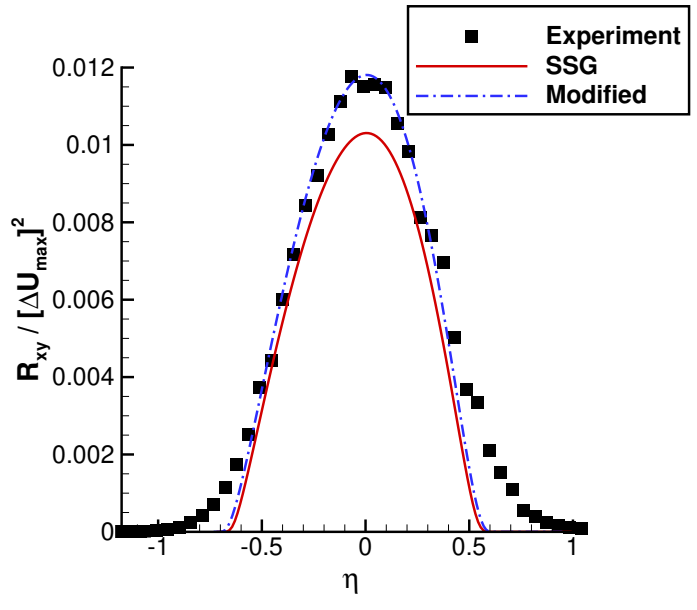

(b) $R_{x y}$ profile.

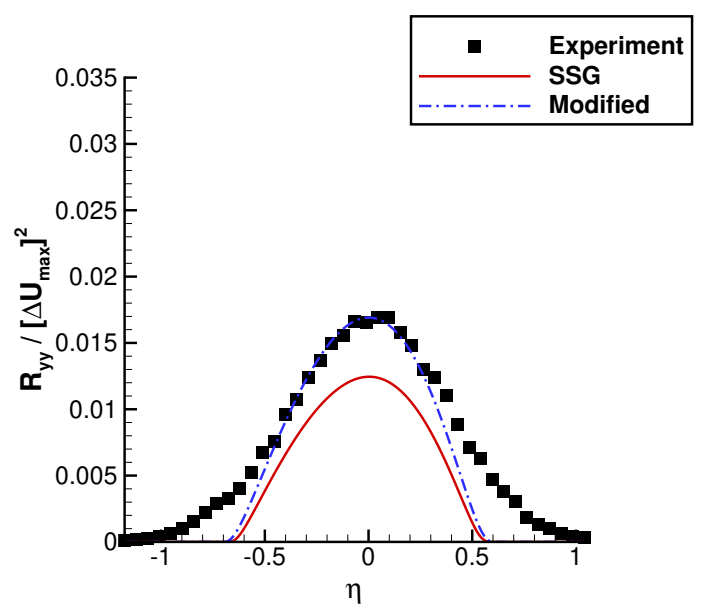

(d) $R_{y y}$ profile.

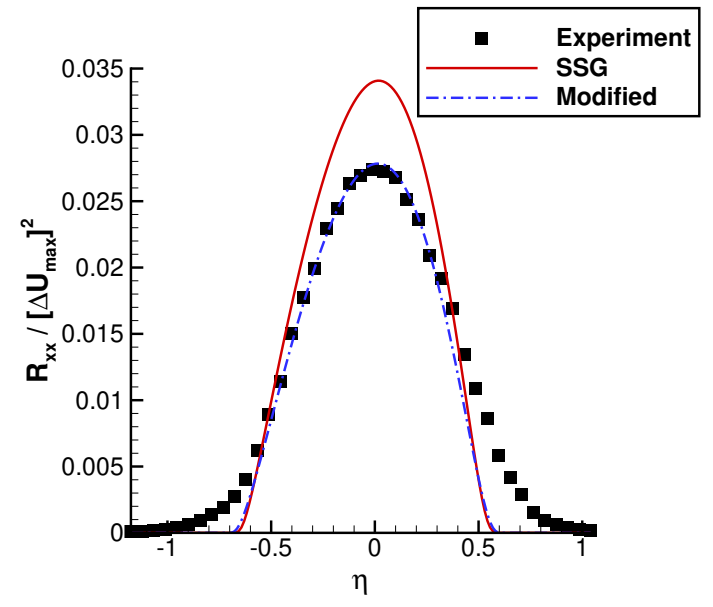

(c) $R_{x x}$ profile.

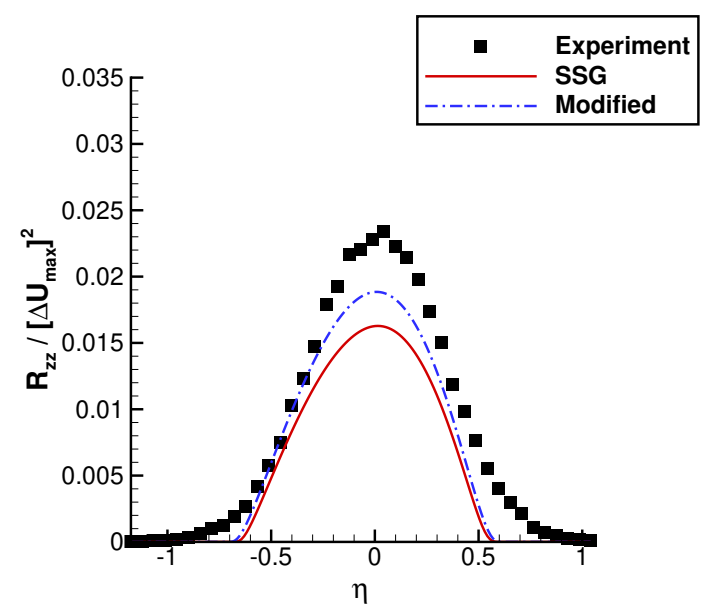

(e) $R_{z z}$ profile.

Fig. 7 Plane mixing layer, mean-velocity and Reynolds-stress profiles. Comparison of Reynolds-stress model predictions with experimental data by Delville et al. [18] at $x=950 \mathrm{~mm}$ downstream of the splitter plate. 


\section{A. Theoretical Profiles}

Based on the assumption of a constant turbulent viscosity, Görtler [5] provides analytical solutions of the averaged incompressible boundary layer equations. These solutions are summarized below for the flows under investigation.

\section{A.A Incompressible Plane Jet}

- Velocity profile

$$
\frac{U}{\Delta U_{\max }(x)}=1-\tanh ^{2} \eta .
$$

- Specific Reynolds-shear stress profile

$$
\frac{R_{x y}}{\left[\Delta U_{\max }(x)\right]^{2}}=\frac{\widehat{S}}{2 \eta_{1 / 2}} \tanh \eta\left(1-\tanh ^{2} \eta\right) .
$$

- Velocity scale: Center line velocity

$$
\Delta U_{\max }(x)=U_{0}(x) .
$$

- Non-dimensional normal coordinate

$$
\eta=\frac{y}{y_{1 / 2}(x)} \eta_{1 / 2} .
$$

- Non-dimensional half-width

$$
\eta_{1 / 2}=\ln (\sqrt{2}+1) \approx 0.881 .
$$

- Spreading rate

$$
\widehat{S}=\frac{d y_{1 / 2}}{d x} .
$$

\section{A.B Incompressible Axisymmetric Jet}

- Velocity profile

$$
\frac{U_{z}}{\Delta U_{\max }(z)}=\frac{1}{\left(\frac{1}{4} \eta^{2}+1\right)^{2}} .
$$

- Specific Reynolds-shear stress profile

$$
\frac{R_{r z}}{\left[\Delta U_{\max }(z)\right]^{2}}=\frac{\widehat{S}}{2 \eta_{1 / 2}} \frac{\eta}{\left(\frac{1}{4} \eta^{2}+1\right)^{3}} .
$$

- Velocity scale: Center line velocity

$$
\Delta U_{\max }(z)=U_{z, 0}(z)
$$


- Non-dimensional radial coordinate

$$
\eta=\frac{r}{r_{1 / 2}(z)} \eta_{1 / 2}
$$

- Non-dimensional half-width

$$
\eta_{1 / 2}=2 \sqrt{\sqrt{2}-1} \approx 1.287
$$

- Spreading rate

$$
\widehat{S}=\frac{d r_{1 / 2}}{d z}
$$

\section{A.C Incompressible Plane Mixing Layer}

Solution for the temporal mixing layer, i. e. in the limit of vanishing velocity difference, $r \rightarrow 1$.

- Velocity profile

$$
\begin{aligned}
\frac{U-U_{m}}{\Delta U_{\max }} & =\frac{1}{\sqrt{\pi}} \int_{0}^{\eta} \exp \left\{-\eta^{\prime 2}\right\} d \eta^{\prime} \\
& =\int_{0}^{\zeta} \exp \left\{-\pi \zeta^{\prime 2}\right\} d \zeta^{\prime} .
\end{aligned}
$$

with mean of bounding velocities

$$
U_{m}=\frac{U_{\max }+U_{\min }}{2}
$$

- Specific Reynolds-shear stress profile

$$
\begin{aligned}
\frac{R_{x y}}{\left[\Delta U_{\text {max }}\right]^{2}} & =\frac{\widehat{S}_{\omega}}{4 \pi} \frac{1+r}{1-r} \exp \left\{\eta^{2}\right\} \\
& =\frac{\widehat{S}_{\omega}}{4 \pi} \frac{1+r}{1-r} \exp \left\{\pi \zeta^{2}\right\}
\end{aligned}
$$

- Velocity scale: Difference of bounding velocities

$$
\Delta U_{\max }=U_{\max }-U_{\min }
$$

- Non-dimensional normal coordinates

$$
\begin{aligned}
\eta & =\sqrt{\pi} \frac{y-y_{m}(x)}{\delta_{\omega}(x)}, \\
\zeta & =\frac{\eta}{\sqrt{\pi}}=\frac{y-y_{m}(x)}{\delta_{\omega}(x)},
\end{aligned}
$$

in which $y_{m}(x)$ denotes the normal coordinate, where $U\left[y_{m}(x)\right]=U_{m}$. 
- Spreading rate

$$
\widehat{S}_{\omega}=\frac{d \delta_{\omega}}{d x} .
$$

The spreading rate can be expressed as

$$
\widehat{S}_{\omega}=\frac{\sqrt{\pi}}{\sigma(r)},
$$

in which

$$
\sigma(r)=\sigma_{0} \frac{1+r}{1-r}
$$

is the spreading parameter that depends on the velocity ratio $r=U_{\max } / U_{\min }$ and $\sigma_{0}$ is the spreading parameter of the half-jet, $r=0$. 